\title{
Adaptive optimal tuning of a general class of stable LTI systems with restricted inputs
}

\author{
H N SHANKAR and K RAJGOPAL \\ Department of Electrical Engineering, Indian Institute of Science, Bangalore \\ 560012 , India \\ e-mail: [shankar,kasi]@ee.iisc.ernet.in \\ MS received 11 January 1996
}

\begin{abstract}
The problem addressed is one of model reference adaptive control (MRAC) of asymptotically stable plants of unknown order with zeros located anywhere in the $s$-plane except at the origin. The reference model is also asymptotically stable and lacking zero(s) at $s=0$. The control law is to be specified only in terms of the inputs to and outputs of the plant and the reference model. For inputs from a class of functions that approach a non-zero constant, the problem is formulated in an optimal control framework. By successive refinements of the sub-optimal laws proposed here, two schemes are finally designed. These schemes are characterized by boundedness, convergence and optimality. Simplicity and total time-domain implementation are the additional striking features. Simulations to demonstrate the efficacy of the control schemes are presented.
\end{abstract}

Keywords. Model reference adaptive control; nonminimum phase; unknown order; optimal control.

\section{Introduction}

By 'control' of a system is meant the process of achieving the desired or close-to-desired performance from the system by manipulating it in some fashion. Often, some simplifying assumptions - linearity, time-invariance, etc. - of the system are made to make the problem more tractable. When the assumptions are too naive or when enhanced performance under varying conditions is required, relatively refined strategies are sought. The built-in capability of a system for such refinement is variously termed adaptation, self-organization, learning and intelligence. An adaptive controller is one which is capable of reconfiguring itself for the 'better', based on its observation of the process as it unfolds.

Two approaches - direct and indirect - have been reported in the literature on adaptive controllers (Astrom 1987). In the indirect approach, the plant is represented by an explicit 
model, the unknown parameters of which are estimated by on-line system identification techniques (Ljung 1987). This requires the knowledge of the plant order or, at any rate, of an upper bound on the plant order. In the absence of knowledge of even a reasonable upper bound on the order, order estimation techniques are resorted to (Sliwa 1984). They are commonly built upon some heuristics, and hence are feasible only in situations where, inter alia, speed of adaptation and plant stability are not critical. In self-tuning regulators, on-line tuning of the controller is achieved through certainty-equivalence. Alternatively, when no explicit effort is made to identify the plant parameters but the controller is directly adjusted to minimize the error between the plant and the reference model outputs, the approach is termed direct model reference adaptive control (MRAC).

Stability of the direct MRAC algorithms is guaranteed in general under the following assumptions (Narendra \& Annaswamy 1989; Sastry \& Bodson 1993)

(i) The plant is minimum phase (zeros restricted to the left half of the $s$-plane);

(ii) the order or an upper bound on the order of the plant is known;

(iii) the reference model is stable and is minimum phase;

(iv) the sign of the high-frequency gain of the plant is known;

(v) the input is persistently exciting.

Assumption (iv) above has been relaxed for a minimum phase plant (Morse 1987). In a recent piece of work (Tao \& Ioannou 1993), one of the basic assumptions in stable MRAC - that the relative degree of the modelled part of the plant is known exactly and is no greater than that of the reference model - is relaxed; the scheme there requires only an upper bound for the relative degree of the plant.

An independent direction recently explored (Bar-Kana et al 1983; Sobel \& Kaufman 1987) for the direct adaptive control problem is the Command Generator Tracker (CGT) method. In this approach (Sobel \& Kaufman 1987), the output error is used to compute the adaptive gains obtained as a combination of 'proportional' and 'integral' terms. The plant command is generated in terms of these adaptive gains. The reference trajectories which the plant output has to follow are limited to the class of outputs of a free-running LTI dynamical system.

Strict positive real (SPR) property is pivotal in several adaptive control schemes. In the CGT approach, for instance, the closed-loop plant being SPR implies asymptotic stability of the system, boundedness of the controller gains and asymptotic tracking. Being highly restrictive, the SPR condition is often relaxed. The plant must then be almost SPR (ASPR) (Narendra \& Annaswamy 1989). If the plant, or an augmented version of it, is ASPR, then a suitable Lyapunov function can be constructed and stability ensured. Else, finding a suitable Lyapunov function cannot, in general, be taken for granted. It is seen that both direct MRAC and CGT approaches call for certain precise a priori structural information about the plant, the order information being one such. In practice, systems frequently have orders much in excess of those assumed for identification. Even if identification were to be the 'best' with respect to the chosen model, the effect of unmodelled dynamics of a linear plant cannot always be totally neglected for persistently exciting inputs. Indeed, all order estimation techniques are prone to be inexact, based as they invariably are, on some heuristic. Despite the existence of rigorous 
stability proofs it has been demonstrated (Rohrs et al 1982, 1985) that several algorithms are non-robust in the presence of 'small' unmodelled dynamics. Hence there is a strong case to develop algorithms which are independent of information of the plant order.

Persistency of excitation, a condition which can be met in practice only by artificial injection of probing signals, is attended with many problems. In several situations, it is simply infeasible to continually inject probing signals. Questions that naturally arise in this context are: Can persistency of excitation be done away with? If yes, what are the issues that need to be addressed as a consequence? What are the associated advantages and disadvantages?

The plant being minimum phase is a rather restrictive, yet vital factor, for stability of adaptive systems as established. Interest in plants which are not necessarily minimum phase has also motivated research. Some of these studies are mentioned below.

- A conceptual approach involving a bilinear parameter estimation problem has been proposed (Astrom 1980; Praly 1984). The procedure (Astrom 1980) is based on identification of an implicit plant model and pole-zero placement design. The estimation requires that at each $t$, a quadratic criterion be minimized (Praly 1984). The assumptions are stabilizability, knowledge of an upper bound on the system order and knowledge of an upper bound on the system parameters.

- A class of discrete time systems inclusive of all minimum phase, all stable nonminimum phase and some unstable nonminimum phase systems has been considered (Goodwin et al 1981). Global stability is assured under the 'key substantive' assumption that the one-step-ahead optimal controller designed using the true (but unknown) system parameters leads to a stable closed-loop system. Closed-loop stability is hypothesized. A modification of the one-step-ahead law to accommodate nonminimum phase zeros has been suggested (Hartley \& Sarantopoulos 1991). Since the procedures involve the estimation of plant parameters, they assume an explicit form for the plant transfer function.

- Loop Transfer Recovery (LTR) as applied to minimum phase plants (Doyle \& Stein 1979,1981 ) has been generalized to nonminimum phase plants (Zhang \& Freudenberg 1990). Necessary and sufficient conditions for a nonminimum phase plant to have a recoverable target loop are arrived at (Chen et al 1992). These procedures involve, inter alia, state observers and a state feedback loop.

Thus the knowledge of the plant order (or an upper bound on the order) is inherent in these approaches.

It is more often a rule than an exception that plants that are minimum phase in continuous time are nonminimum phase in their discrete time approximate representations. If an upper bound on the plant order is unknown, reported studies of plants with nonminimum phase zeros are inapplicable. Moreover, when the plant is nonminimum phase, matching of the closed-loop transfer function with the transfer function of the reference model is impossible for persistently exciting inputs (except possibly in the trivial case when all nonminimum phase zeros of the unknown plant and the reference model have exactly matching locations and corresponding orders). This is because a nonminimum phase plant does not have a stable inverse. Consequently, adaptive tuning of systems which are allowed 
to be nonminimum phase must be over some constrained class of inputs if asymptotic exact model following is demanded.

There is a good practical reason (Benes 1965) to restrict the inputs to the Marcinkiewicz Space, $\mathcal{M}_{2}$ (Benes 1965), the space of bounded functions of bounded power.

$$
\begin{aligned}
\mathcal{M}_{2}= & \left\{x(\cdot):[0, \infty) \longmapsto \Re: \int_{0}^{T}|x(t)|^{2} \mathrm{~d} t<\infty \forall T \in \mathfrak{H}^{+},\right. \\
& \left.\limsup _{T \rightarrow \infty} \frac{1}{T} \int_{0}^{T}|x(t)|^{2} \mathrm{~d} t<\infty\right\} .^{*}
\end{aligned}
$$

$\mathcal{L}_{2} \subset \mathcal{M}_{2}$. In particular, the step and the sinusoids belong to $\mathcal{M}_{2}$. It is significant here to observe that in the CGT approach the reference trajectories which the plant output has to follow are restricted to the class of outputs of a free-running LTI dynamical system.

The main features of this presentation constitute relaxing the three usual requirements as follows.

(a) No a priori information is needed about the parameters or the order or relative degree of the plant;

(b) the plant is allowed to be nonminimum phase;

(c) the input is not necessarily persistently exciting.

However, the demand is that the plant be asymptotically stable. An additional mild restriction is that the plant and the reference model shall have no zero(s) at $s=0$.

This paper is organized as follows. The problem is formulated in $\S 2$. Section 3 addresses amplitude matching, in part, and sign matching of the outputs. Two optimal schemes are proposed in $\S 4$. Simulations comprise $\S 5$.

\section{Problem formulation}

In this section, we formally state the problem, state some preliminary results and cast the problem in an optimization framework. Alongside the process of choosing a criterion to be minimized, we show how it captures all aspects of the problem. Our approach to solving the problem will also be indicated.

\subsection{The statement of the problem}

The MRAC problem addressed here involves finite-dimensional, LTI, SISO systems. The given plant $G_{p}(s)$ is such that

- (A1) its order or even an upper bound on its order is unknown;

- (A2) it may be nonminimum phase;

$\overline{*^{+}+(0, \infty)}$. 
- (A3) it is asymptotically stable;

- (A4) it is strictly proper and

- (A5) it has no zero(s) at $s=0$.

The reference model, fully specified by a transfer function $G_{m}(s)$, satisfies (A2) (A5). The nonminimum phase zero(s) of the plant and the model need not have matching location(s).

It is often desired to match, or approximate as closely as possible, the step responses of the plant to be controlled and the reference model. We allow the reference model inputs to belong to a class of functions that are 'step-like'; i.e., a class of functions that asymptotically approach a constant. We define this class of 'step-like' functions, to which the reference model inputs belong. ${ }^{\dagger}$

\section{DEFINITION 1}

$\Omega_{u, f} \triangleq\{u:[0, \infty) \longmapsto \mathfrak{R}\}$ such that $u(t)$

(1) is bounded and is either continuous or has finitely many discontinuities of the first kind;

(2) is differentiable as $t \rightarrow \infty$ and the limit of the time derivative is zero;

(3) has a non-zero limit (which is finite by virtue of 1 and 2) as $t \rightarrow \infty$.

It is required to specify an on-line adaptive scheme which ensures convergence of the controller so that the plant output matches or approximates the model output. Also, boundedness of the controller must be assured.

\subsection{Certain preparatory results}

\section{PROPOSITION 1}

Let $f_{1}:[0, \infty) \longmapsto \Re$ be continuous or have utmost finitely many discontinuities of the first kind.

(1) If $\lim _{t \rightarrow \infty} f_{1}(t)=c$, then $\lim _{T \rightarrow \infty}(1 / T) \int_{0}^{T} f_{1}(\tau) \mathrm{d} \tau=c$.

(2) $\int_{0}^{t} f_{1}(\tau) \mathrm{d} \tau$ is continuous for $t \in[0, \infty)$.

\section{PROPOSITION 2}

If $f_{1}, f_{2}:[0, \infty) \longmapsto \Re$ satisfy $\int_{0}^{\infty} f_{1}^{2}(\tau) \mathrm{d} \tau>0$ and $\int_{0}^{\infty} f_{2}^{2}(\tau) \mathrm{d} \tau>0$, then

$$
\lim _{t \rightarrow \infty}\left[(1 / t) \int_{0}^{t} f_{1}^{2}(\tau) \mathrm{d} \tau\right] /\left[(1 / t) \int_{0}^{t} f_{2}^{2}(\tau) \mathrm{d} \tau\right] \text { exists (it may be infinite). }
$$

Proof. Letting $N(t) \triangleq \int_{0}^{t} f_{1}^{2}(\tau) d \tau$ and $D(t) \triangleq \int_{0}^{t} f_{2}^{2}(\tau) d \tau$, the required limit is $\lim _{t \rightarrow \infty}$ $[N(t) / D(t)] . N(t)$ and $D(t)$ are nonnegative and nondecreasing; hence $\lim _{t \rightarrow \infty} N(t)$ and

\footnotetext{
${ }^{\dagger}$ The scope of this paper is restricted to inputs of this class. Results pertaining to inputs comprising signals that approach a sinusoid without and with a dc offset have also been obtained. In fact, the subscript $f$ for 'final value' in $\Omega_{u, f}$ is meant to differentiate this class from $\Omega_{u, s}$ and $\Omega_{u, c}$, the classes of functions which respectively approach a 'sinusoid' without an offset and a sinusoid with an offset ('combination').
} 
$\lim _{t \rightarrow \infty} D(t)$ exist. If both limits are finite or if only one of them is infinite, the proposition is proved. Else, the required limit has the form $\infty / \infty$. If as $t \rightarrow \infty$, the order at which $N(t) \rightarrow \infty$ is higher than (the same as) [lower than] that at which $D(t) \rightarrow \infty$, then the required limit is infinite (positive and finite) [zero].

Lemma 1. If $u \in \Omega_{u, f}$ be the input and $y$ the output of a system $\tilde{T}(s)$ satisfying (A1)(A4), then

$$
\lim _{t \rightarrow \infty}\left|\left\{\frac{1}{t} \int_{0}^{t} y^{2}(\tau) \mathrm{d} \tau\right\}^{\frac{1}{2}}\right|=\left|M_{\infty} \tilde{T}(0)\right| \quad \text { where } M_{\infty} \triangleq \lim _{t \rightarrow \infty} u(t) .
$$

Proof. By (A3) and (A4), $\widetilde{T}(0)$ is finite and $\tilde{T}(s)$ has a state model

$$
\dot{x}(t)=A x(t)+B u(t), \quad y(t)=C x(t),
$$

with $\tilde{T}(s)=C(s I-A)^{-1} B$ and $\lim _{t \rightarrow \infty} C e^{A t} B=0$. The zero-state response is $y(t)=\int_{0}^{t} C e^{A(t-\tau)} B u(\tau) \mathrm{d} \tau$. Since $u \in \Omega_{u, f}, C e^{A(t-\tau)} B u(\tau)$ has utmost finitely many discontinuities of the first kind. Then by part 2 of proposition $1 y$ is continuous. Clearly, $y$ is bounded and $\lim _{t \rightarrow \infty} y(t)=\widetilde{T}(0) M_{\infty}$. By applying part 1 of proposition 1 to $y^{2}$,

$$
\lim _{t \rightarrow \infty} \frac{1}{t} \int_{0}^{t} y^{2}(\tau) \mathrm{d} \tau=\widetilde{T}^{2}(0) M_{\infty}^{2}
$$

and the lemma follows.

\subsection{The performance index}

We use the foregoing results in constructing a performance index and provide an insight into its significance as we do so.

Let $g_{m}$ and $g_{p}$ respectively denote the impulse responses of the reference model $G_{m}(s)$ and the unknown plant $G_{p}(s)$. Further, let $u_{m} \in \Omega_{u, f}$. The model output $y_{m}(t)=\int_{0}^{t} g_{m}(t-$ $\tau) u_{m}(\tau) \mathrm{d} \tau$. As even an upper bound on the order of $G_{p}(s)$ is not known, we are left with a situation wherein $u_{m}, y_{m}$ and $y_{p}$ are the only functions available to generate the plant command $u_{p}$. Thus, $y_{p}(t)=\int_{0}^{t} g_{p}(t-\tau) u_{p}(\tau) \mathrm{d} \tau$, where $u_{p}(t)=h\left(u_{m}, y_{m}, y_{p}, t\right)$.

As the aim is to specify $h$ such that $y_{p}$ matches or approximates $y_{m}$, the error $\left\{y_{m}-y_{p}\right\}$ deserves to be incorporated into the performance index. Since error-minimization is sought over the range, $[0, \infty)$, of time, $\int_{0}^{\infty}\left\{y_{m}(\tau)-y_{p}(\tau)\right\}^{2} \mathrm{~d} \tau$ may serve as a performance index. However, this would tend to infinity with even a mild mismatch between $y_{m}(t)$ and $y_{p}(t)$ as $t \rightarrow \infty$.

Consider

$$
\begin{aligned}
\int_{0}^{\infty}\left\{y_{m}(\tau)-y_{p}(\tau)\right\}^{2} \mathrm{~d} \tau= & \int_{0}^{\infty} y_{m}^{2}(\tau) \mathrm{d} \tau+\int_{0}^{\infty} y_{p}^{2}(\tau) \mathrm{d} \tau \\
& -2 \int_{0}^{\infty} y_{m}(\tau) y_{p}(\tau) \mathrm{d} \tau .
\end{aligned}
$$

Even for a step input, $\int_{0}^{\infty} y_{m}^{2}(\tau) \mathrm{d} \tau=\infty$. However, in the light of lemma 1, it is clear that when $u_{m} \in \Omega_{u, f}$, 


$$
\lim _{t \rightarrow \infty} \frac{1}{t} \int_{0}^{t} y_{m}^{2}(\tau) \mathrm{d} \tau=G_{m}^{2}(0)\left\{\lim _{t \rightarrow \infty} u_{m}(t)\right\}^{2} \in(0, \infty),
$$

since $G_{m}(s)$ is asymptotically stable and without any zero at $s=0$ and $\lim _{t \rightarrow \infty} u_{m}(t)$ is non-zero and finite. If $u_{p} \in \Omega_{u, f}$, then by lemma 1 in like fashion,

$$
\lim _{t \rightarrow \infty} \frac{1}{t} \int_{0}^{t} y_{p}^{2}(\tau) \mathrm{d} \tau=G_{p}^{2}(0)\left\{\lim _{t \rightarrow \infty} u_{p}(t)\right\}^{2} \in(0, \infty)
$$

By proposition 1 it can be shown that

$$
\lim _{t \rightarrow \infty} \frac{1}{t} \int_{0}^{t} y_{m}(\tau) y_{p}(\tau) \mathrm{d} \tau=G_{m}(0) G_{p}(0)\left\{\lim _{t \rightarrow \infty} u_{m}(t)\right\}\left\{\lim _{t \rightarrow \infty} u_{p}(t)\right\},
$$

which also is finite and non-zero. Hence the performance index would be more encompassing if it comprised

$$
\lim _{t \rightarrow \infty} \frac{1}{t} \int_{0}^{t}\left\{y_{m}(\tau)-y_{p}(\tau)\right\}^{2} \mathrm{~d} \tau
$$

This is the mean-square disparity between $y_{m}$ and $y_{p}$. Evidently, being a function of $u_{m}$, it will, in general, be different for different model inputs of $\Omega_{u, f}$. To facilitate cost comparison for different inputs, the candidate performance index can be refined to

$$
\overline{J_{f}} \triangleq\left[\lim _{t \rightarrow \infty} \frac{1}{t} \int_{0}^{t}\left\{y_{m}(\tau)-y_{p}(\tau)\right\}^{2} \mathrm{~d} \tau\right] /\left[\lim _{t \rightarrow \infty} \frac{1}{t} \int_{0}^{t} y_{m}^{2}(\tau) \mathrm{d} \tau\right] .
$$

Lemma 1 shows that the denominator of $\overline{J_{f}}$ is positive and finite. We may therefore specify $\overline{J_{f}}$ as a well-formed criterion for minimization. Nonetheless, it has the limitation that all inputs belonging to $\Omega_{u, f}$ are not concurrently considered. With a view to minimize the worst error as in $\mathcal{H}^{\infty}$-optimal control the performance index is chosen as:

$$
J_{f} \triangleq \sup _{u_{m} \in \Omega_{u, f}}\left\{d\left(u_{m}\right): d\left(u_{m}\right)=\frac{\lim _{t \rightarrow \infty}(1 / t) \int_{0}^{t}\left\{y_{m}(\tau)-y_{p}(\tau)\right\}^{2} \mathrm{~d} \tau}{\lim _{t \rightarrow \infty}(1 / t) \int_{0}^{t} y_{m}^{2}(\tau) \mathrm{d} \tau}\right\} .
$$

The goal is to generate $u_{p}$ that minimizes $J_{f}$. Observe that $J_{f}$ is bounded below by zero.

\subsection{The approach to solution}

$u_{m}, y_{m}$ and $y_{p}$ are the only signals available to specify the plant command, $u_{p}$, as in figure 1. The adaptive controller in figure 1 represents a nonlinear time-varying gain $h$. For $u_{m} \in \Omega_{u, f}, h$ has to settle at a value whose magnitude and sign assure matching of the magnitude and sign of $y_{m}$ by those of $y_{p}$. Boundedness of $h$ is a sine qua non.

\section{Suboptimal schemes}

We shall gradually build the optimal control law starting from two suboptimal schemes in this section. This is because the suboptimal schemes provide an insight into the various aspects of the problem arising out of 


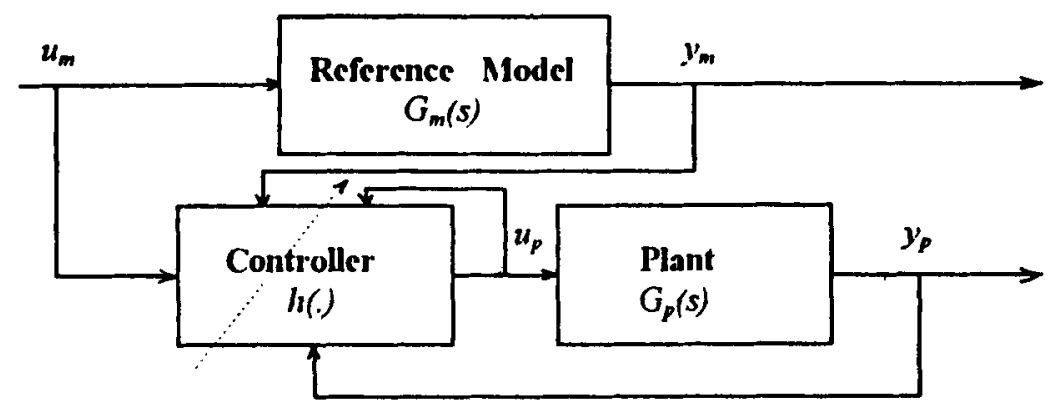

Figure 1. A schematic of the setup.

- relaxing the assumptions on the plant, viz., information about its order and its being minimum or nonminimum phase; and

- the reference model inputs belonging to $\Omega_{u, f}$.

The following observation is pertinent before we set out to present the suboptimal laws.

Observation 1. It was emphasized in the previous section that $u_{m}, y_{m}$ and $y_{p}$ are the only signals in terms of which the plant command, $u_{p}$, has to be specified. Moreover, $J_{f}$ of (1) is independent of any parameter of the plant or model or otherwise. The problem is therefore not in the class of parametric optimization problems. Consequently, we cannot resort to methods such as gradient descent in some parametric space. This will lead us to laws and proofs that are interesting because of their unconventional approach, as will be demonstrated.

Lemma 2 (below) throws light on the aspect of on-line matching, in magnitude, of the model output by the plant output. On-line matching of signs of the model and the plant outputs is ensured by lemma 3 . Lemma 4 is a variant of lemma 3 and it achieves the same purpose.

In what follows in this and the later sections, unless otherwise stated, it is understood that we carry forward the notations and specifications of $\S 2$. Further, zero initial conditions are assumed. This assumption will be relaxed in corollary 2 towards the end of $\S 4$.

We are interested in the matching of $y_{m}$ by $y_{p}$ for the 'step-like' inputs. It will be instructive to explore the different candidates for the map $h$ of figure 1 by considering an example.

Example 1. Let $G_{m}(0)=4$ and $G_{p}(0)=-1$. Then with $u_{m}(t)$ a unit step, $\lim _{t \rightarrow \infty} y_{m}(t)$ $=4$ and $\lim _{t \rightarrow \infty} y_{p}(t)=-1$. In the control law of the form $u_{p}(t)=h(t) u_{m}(t)$, if an $h$ satisfying $\lim _{t \rightarrow \infty} h(t)=-4$ were chosen, we can achieve the desired matching asymptotically. Toward this end, as a candidate for $h$, suppose we set $h_{1}(t) \triangleq y_{m}(t) / y_{p}(t)$. A little consideration will show that this may lead to unboundedness of the controller though $y_{m}$ is bounded. This is because it is attended by the problem of zero-crossings of $y_{p}$ due to:

(i) $G_{p}(s)$ being allowed to be nonminimum phase with unknown zero locations; and

(ii) zero-crossings of $u_{m}(t)$ for any finite $t$ are permitted by $\Omega_{u, f}$. 
An $h_{2}(t)$ of the form $\int_{0}^{t} y_{m} / \int_{0}^{t} y_{p}$ also suffers from the same drawback. To circumvent such problems, we may as well look at a map $h$ with its denominator positive and nondecreasing, say. Then,

$$
h_{3}^{2}(t) \triangleq \int_{0}^{t} y_{m}^{2} / \int_{0}^{t} y_{p}^{2} .
$$

In computing $h_{3}$ from the above, the loss of sign information is evident. Nonetheless, putting off, for the present, the issue of sign-matching, we proceed by taking the cue from this last form, $h_{3}$, and investigate amplitude matching.

Lemma 2. In the setup of figure 1 , let $u_{p}(\cdot) \triangleq \alpha(\cdot) u_{m}(\cdot)$ where $u_{m} \in \Omega_{u, f}$ and

$$
\alpha(t) \triangleq\left\{\begin{array}{c}
1 ; 0 \leq t \leq T_{1}<\infty \\
\quad \exists t_{1} \in\left(0, T_{1}\right) \ni y_{m}\left(t_{1}\right) y_{p}\left(t_{1}\right) \neq 0 \\
\left|\left\{\left[\frac{1}{t} \int_{0}^{t} y_{m}^{2}(\tau) d \tau\right] /\left[\frac{1}{t} \int_{0}^{t} y_{p}^{2}(\tau) d \tau\right]\right\}^{\frac{1}{2}}\right| ; t>T_{1} .
\end{array}\right.
$$

Then

(1) $\alpha$ is bounded;

(2) $\lim _{t \rightarrow \infty} \alpha(t)=\left|\left\{\left|G_{m}(0) / G_{p}(0)\right|^{\frac{1}{2}}\right\}\right|$.

Note. Intermediate steps in some proofs are indicated by subtitles.

A rigorous proof of this lemma has been worked out (Shankar 1993). We sketch here an outline of that proof with some details here and there.

Proof. Denote $\lim _{t \rightarrow \infty} u_{m}(t)=M_{m}$. Then $\lim _{t \rightarrow \infty} y_{m}(t)=G_{m}(0) M_{m}$, and as $G_{m}(s)$ has no zero at $s=0$, by part 1 of proposition 1 ,

$$
\lim _{t \rightarrow \infty} \frac{1}{t} \int_{0}^{t} y_{m}^{2}(\tau) \mathrm{d} \tau=G_{m}^{2}(0) M_{m}^{2} \in(0, \infty) .
$$

Claim. $\alpha(t), t \in[0, \infty)$, is finite.

The boundedness of $y_{m}$ and the definition of $T_{1}$ in (2) is pivotal in proving this claim. $T_{1}$ is a time until after the plant and model outputs are excited. It is necessitated by the definition of $\Omega_{u, f}$ which allows $u_{m}$ to be zero except at removable discontinuities for some finite time. It serves an additional nontrivial purpose; that will find mention in the section on simulations.

Claim. $\lim _{t \rightarrow \infty} \alpha(t)$ exists.

Follows from suitably applying proposition 2 to (2).

Our next aim will be to show that $\alpha$ settles to a positive, finite value.

Claim. $\quad \lim _{t \rightarrow \infty} \alpha(t) \neq \infty$.

Suppose $\lim _{t \rightarrow \infty} \alpha(t)=\infty$. Then $\left|\lim _{t \rightarrow \infty} u_{p}(t)\right|=\infty$. Two cases arise here. 
Case 1. $\lim _{t \rightarrow \infty}\left|u_{p}(t)\right|=\infty$ such that $\lim _{t \rightarrow \infty}\left|y_{p}(t)\right|<\infty$.

As $G_{p}(s)$ may be nonminimum phase, it is necessary to consider a situation wherein $u_{p}$ may go unbounded such that $y_{p}$ remains bounded. This is possible iff each factor of the form $\left(s-\sigma_{i}\right), \sigma_{i}>0$, in the denominator of $U_{p}(s)$ is cancelled by a corresponding nonminimum phase zero, $\left(s-\sigma_{i}\right)$, of $G_{p}(s)$. Consequently, $\alpha(t)$ has to grow exponentially resulting in $\lim _{t \rightarrow \infty}\left(\alpha^{2}(t) / t\right)=\infty$ and thence,

$$
\lim _{t \rightarrow \infty} \alpha^{2}(t) \frac{1}{t} \int_{0}^{t} y_{p}^{2}(\tau) \mathrm{d} \tau=\infty
$$

The LHS of the above is also, by virtue of (2) and (3),

$$
\lim _{t \rightarrow \infty} \frac{1}{t} \int_{0}^{t} y_{m}^{2}(\tau) \mathrm{d} \tau=G_{m}^{2}(0) M_{m}^{2}<\infty .
$$

Contradiction! Hence

$$
\left\{\lim _{t \rightarrow \infty} \alpha(t)<\infty\right\} \text { OR }\left\{\lim _{t \rightarrow \infty} \alpha(t)=\infty \text { and } \lim _{t \rightarrow \infty}\left|y_{p}(t)\right|=\infty\right\} .
$$

The first option proves the claim. The second leads to the following case.

Case 2. $\lim _{t \rightarrow \infty}\left|u_{p}(t)\right|=\infty$ such that $\lim _{t \rightarrow \infty}\left|y_{p}(t)\right|=\infty$.

Here, it can be shown by invoking (2) and (3), $\lim _{t \rightarrow \infty} \alpha(t)<\infty$, thus violating the supposition that $\lim _{t \rightarrow \infty} \alpha(t)=\infty$.

Hence the claim.

Part 1 of the lemma, namely, the boundedness of $\alpha$ now directly follows. Clearly, therefore, $u_{p}$ and hence $y_{p}$ are bounded too.

Claim. $\lim _{t \rightarrow \infty} \alpha(t) \neq 0$.

Suppose not. Then $\lim _{t \rightarrow \infty} u_{p}(t)=0$ and, by lemma $1, \lim _{t \rightarrow \infty}(1 / t) \int_{0}^{t} y_{p}^{2}(\tau) \mathrm{d} \tau=0$. Using (3) in (2),

$$
\lim _{t \rightarrow \infty} \alpha^{2}(t)=\lim _{t \rightarrow \infty}\left\{\left[\frac{1}{t} \int_{0}^{t} y_{m}^{2}(\tau) \mathrm{d} \tau\right] /\left[\frac{1}{t} \int_{0}^{t} y_{p}^{2}(\tau) \mathrm{d} \tau\right]\right\}=\infty,
$$

a contradiction. The claim is established.

Remark 1. In fact, this boot-strapping property of $\alpha(t)$ for $t>T_{1}$, as demonstrated in the proof till now, is one of the motivations behind the definition of $\alpha$ as in (2).

Remark 2. As $\alpha$ and $u_{m}$ are bounded, an examination of $\int_{0}^{t} y_{m}^{2}(\tau) \mathrm{d} \tau$ and $\int_{0}^{t} y_{p}^{2}(\tau) \mathrm{d} \tau$ reveals, in view of part 2 of proposition 1 , that $\alpha(t)$ is continuous in $t$, except possibly at $t=T_{1}$.

Claim. $\lim _{t \rightarrow \infty}(\mathrm{d} \alpha(t) / \mathrm{d} t)=0$.

In view of the remark $2, u_{p}=\alpha u_{m}$ is bounded and has utmost finitely many discontinuities of the first kind. Part 2 of proposition 1 then establishes that $y_{m}$ and $y_{p}$ are continuous. Thus $\alpha(t)$ is differentiable w.r.t. $t, t>T_{1}$. Then by virtue of $\lim _{t \rightarrow \infty} \alpha(t) \in(0, \infty)$, the claim holds. 
Evaluation of $\lim _{t \rightarrow \infty} \alpha(t)$ : Let $\alpha_{f} \triangleq \lim _{t \rightarrow \infty} \alpha(t)$. Then $\lim _{t \rightarrow \infty} u_{p}(t)=\alpha_{f} M_{m}$. From the properties of $\alpha$ proved hitherto, it can be shown that $u_{p} \in \Omega_{u, f}$. Hence by lemma 1 applied to $G_{p}(s)$ excited by $u_{p}$,

$$
\lim _{t \rightarrow \infty}\left|\left\{\frac{1}{t} \int_{0}^{t} y_{p}^{2}(\tau) \mathrm{d} \tau\right\}^{\frac{1}{2}}\right|=\left|G_{p}(0) \alpha_{f} M_{m}\right|>0 .
$$

Using this and (3) in (2) and simplifying,

$$
\alpha_{f}=\left|\left\{\left|\frac{G_{m}(0)}{G_{p}(0)}\right|^{\frac{1}{2}}\right\}\right|
$$

With a small technical modification in lemma 2, Corollary 1 follows directly.

\section{COROLLARY 1}

The results of lemma 2 hold with $\alpha(\cdot)$ redefined as

$$
\alpha(t) \triangleq\left\{\begin{array}{c}
1 ; 0 \leq t \leq T_{1}+\varepsilon<\infty, T_{1}, \varepsilon>0 \\
\exists t_{1} \in\left(0, T_{1}\right) \ni y_{m}\left(t_{1}\right) y_{p}\left(t_{1}\right) \neq 0 \\
\left|\left\{\left[\frac{1}{t+\varepsilon} \int_{0}^{t-\varepsilon} y_{m}^{2}(\tau) \mathrm{d} \tau\right] /\left[\frac{1}{t+\varepsilon} \int_{0}^{t-\varepsilon} y_{p}^{2}(\tau) \mathrm{d} \tau\right]\right\}^{\frac{1}{2}}\right| ; t>T_{1}+\varepsilon
\end{array}\right.
$$

Remark 3. Motivated by the discussion in example 1 , in corollary 1 a law was proposed which, though blind to $\operatorname{sgn}\left\{G_{m}(0)\right\}$ vis-a-vis $\operatorname{sgn}\left\{G_{p}(0)\right\}$, was meant to ensure on-line magnitude-matching. But such gain-matching is actually not achieved by corollary 1 as

- $\alpha_{f}=2$, and not 4 as is required for gain-matching, and

- $\lim _{t \rightarrow \infty}\left|y_{m}(t)\right|=\lim _{t \rightarrow \infty}\left|y_{p}(t)\right|$ requires $\lim _{t \rightarrow \infty} \alpha(t)=1$. This is obvious from (2) seen in the light of lemma 1.

Clearly, unless $\left|G_{m}(0)\right|=\left|G_{p}(0)\right|$, corollary 1 does not ensure gain-matching in magnitude. Moreover, if $\left|G_{m}(0)\right|=\left|G_{p}(0)\right|$, such gain-matching is itself superfluous. In other words, if in corollary $1, \lim _{t \rightarrow \infty} \alpha(t)$ equals unity, $\alpha$ itself can be dispensed with from the control law! Nonetheless, the control $u_{p}$ of corollary 1 is a step towards dc gain-matching. This is because the insight gained by analyzing the shortcomings of corollary 1 will be exploited in the next section in the design of an optimal controller. In addition, the proof of lemma 2, which is essentially the same as that of corollary 1 , simplifies the proof of theorem 1 (see § 4) as well.

Remark 4. More importantly, the control of corollary 1 is insensitive to $\operatorname{sgn}\left\{G_{m}(0)\right\}$ vis-avis $\operatorname{sgn}\left\{G_{p}(0)\right\}$. In handling plants and reference models which are allowed to be nonminimum phase this further inadequacy of the control law of corollary 1 is obvious. Lemma 3 is meant to show how sign-matching can be achieved by introducing an additional factor $\beta:[0, \infty) \longmapsto\{1,-1\}$ into the law given by corollary 1 . 
Lemma 3. In the setup of figure 1, if $u_{p}(\cdot) \triangleq \alpha(\cdot) \beta(\cdot) u_{m}(\cdot)$ where $\alpha$ is given by corollary 1 and

$$
\beta(t) \triangleq\left\{\begin{aligned}
1 ; \quad & 0 \leq t \leq T_{\beta}, \quad T_{\beta} \in\left(T_{1}, \infty\right) \\
-1 ; & 2^{i} T_{\beta}<t \leq 2^{i+1} T_{\beta}, u_{p}\left(2^{i} T_{\beta}\right) \cdot y_{p}\left(2^{i} T_{\beta}\right) G_{m}(0)<0 \\
& i=0,1,2, \ldots ; \\
1 ; & 2^{i} T_{\beta}<t \leq 2^{i+1} T_{\beta}, u_{p}\left(2^{i} T_{\beta}\right) \cdot y_{p}\left(2^{i} T_{\beta}\right) G_{m}(0) \geq 0 \\
& i=0,1,2, \ldots
\end{aligned}\right.
$$

then

(1) $u_{p}$ and $y_{p}$ are bounded;

(2) $\lim _{t \rightarrow \infty}\left|u_{p}(t)\right|=\left|\left\{\left|G_{m}(0) / G_{p}(0)\right|\right\}^{\frac{1}{2}}\right|\left|M_{m}\right|$, where $M_{m} \triangleq \lim _{t \rightarrow \infty} u_{m}(t)$;

(3) $\lim _{t \rightarrow \infty}\left|y_{p}(t)\right|=\left|\left\{\left|G_{m}(0) G_{p}(0)\right|\right\}^{\frac{1}{2}}\right| \cdot\left|M_{m}\right|$;

(4) the performance index

$$
J_{f}=\left[G_{m}^{2}(0)+\left|G_{m}(0) G_{p}(0)\right|-2\left|\left\{\left|G_{m}(0)\right|^{\frac{3}{2}} \cdot\left|G_{p}(0)\right|^{\frac{1}{2}}\right\}\right|\right] / G_{m}^{2}(0) .
$$

A comprehensive proof of this lemma has been worked out (Shankar 1993). Only an abridged version thereof will be presented here to give a flavour of the nature of the issues involved and the methods employed to address them.

Proof. As seen in the proof of lemma 2 (vide (3)),

$$
\lim _{t \rightarrow \infty} \frac{1}{t} \int_{0}^{t} y_{m}^{2}(\tau) \mathrm{d} \tau=G_{m}^{2}(0) M_{m}^{2} \in(0, \infty)
$$

Claim. $\alpha, u_{p}$ and $y_{p}$ are bounded and $\lim _{t \rightarrow \infty} \alpha(t)$ exists.

This follows by suitably adapting the proof of lemma 2 and is regardless of the existence of $\lim _{t \rightarrow \infty} \beta(t)$. It is important in this context to observe that $\beta= \pm 1$ and is allowed to change only at discrete instants of time separated by exponentially growing intervals. This is part 1 of the lemma.

The introduction of $\beta= \pm 1$ into the control law here does not require more than straightforward modifications in the proof of lemma 2 in establishing that $\lim _{t \rightarrow \infty} \alpha(t) \neq 0$ and

$$
\lim _{t \rightarrow \infty} \mathrm{d} \alpha(t) / \mathrm{d} t=0
$$

Claim. $\lim _{t \rightarrow \infty} \beta(t)$ exists and equals $\operatorname{sgn}\left\{G_{m}(0) G_{p}(0)\right\}$.

Herein lies the focus of this lemma. By definition $\beta(t)$ is constant for $t \in\left(2^{i} T_{\beta}, 2^{i+1} T_{\beta}\right]$, $i=0,1,2 \ldots$. The interval $\left(2^{i+1}-2^{i}\right) T_{\beta}$ increases without bound as $i \rightarrow \infty$. Let $t_{l \beta}$ and $t_{u \beta}$ be such that for some $i, 2^{i} T_{\beta}<t_{l \beta}<t_{u \beta} \leq 2^{i+1} T_{\beta}$. Suppose

$$
\beta(t)=+1, t \in\left[t_{l \beta}, t_{u \beta}\right] \text {. }
$$


Then for $t \in\left[t_{l \beta}, t_{u \beta}\right]$,

$$
u_{p}(t)=\alpha(t) u_{m}(t)
$$

As $u_{m} \in \Omega_{u, f}$ is differentiable as $t \rightarrow \infty, \exists t_{\beta 1} \in \mathfrak{R}^{+} \ni \dot{u}_{m}(t)$ exists $\forall t>t_{\beta 1}$. Choose

$$
t_{l \beta} \geq t_{\beta 1} \text {. }
$$

Then $\dot{u}_{m}(t)$ exists $\forall t>t_{l \beta}$. So from (7), for $t \in\left(t_{l \beta}, t_{u \beta}\right]$, the interval in which $\beta$ is not allowed to change,

$$
\dot{u}_{p}(t)=\dot{\alpha}(t) u_{m}(t)+\alpha(t) \dot{u}_{m}(t)
$$

Assuming for the moment that (7) holds for all $t \geq t_{l \beta},(9)$ yields, in view of (5), $\lim _{t \rightarrow \infty} \dot{u}_{p}$ $(t)=0$

$$
\text { i.e., for any } \varepsilon_{\beta 1}>0, \exists t_{\beta 2} \in \mathfrak{R}^{+} \ni\left|\dot{u}_{p}(t)\right|<\varepsilon_{\beta 1} \forall t>t_{\beta 2} \text {. }
$$

Let

$$
t_{l \beta} \geq t_{\beta 2}
$$

Note that even though (7) does not necessarily hold for all $t \geq t_{l \beta}$, but only for $t \in\left[t_{l \beta}, t_{u \beta}\right]$, yet in view of (11),

$$
\left|\dot{u}_{p}(t)\right|<\varepsilon_{\beta 1}, t_{l \beta} \leq t \leq t_{u \beta} .
$$

This is because of the causal nature of the system. Equation (12) was derived primarily to illustrate how system causality can be used to advantage in proving the existence of $\lim _{t \rightarrow \infty} \beta(t)$.

Now back to the assumption that (7) holds for all $t \geq t_{1 \beta}$,

$$
\begin{aligned}
y_{p}(t)=\int_{0}^{t} g_{p}(t-\tau) u_{p}(\tau) \mathrm{d} \tau=\int_{0}^{t_{l \beta}} & C_{p} e^{A_{p}(t-\tau)} B_{p} u_{p}(\tau) \mathrm{d} \tau \\
& +\int_{t_{l \beta}}^{t} C_{p} e^{A_{p}(t-\tau)} B_{p} u_{p}(\tau) \mathrm{d} \tau
\end{aligned}
$$

$\left(C_{p}, A_{p}, B_{p}\right)$ is a minimal realization of $G_{p}(s)$. As $\lim _{t \rightarrow \infty} e^{A_{p} t}=\mathbf{0}$,

$$
\begin{array}{r}
\lim _{t \rightarrow \infty} \int_{0}^{t_{l \beta}} C_{p} e^{A_{p}(t-\tau)} B_{p} u_{p}(\tau) \mathrm{d} \tau=\int_{0}^{t_{l \beta}} C_{p} \cdot \lim _{t \rightarrow \infty}\left[e^{A_{p}(t-\tau)}\right] B_{p} u_{p}(\tau) \mathrm{d} \tau=0 . \\
\text { i.e., for any } \varepsilon_{\beta 2}>0, \exists t_{\beta 3} \in \Re^{+} \ni\left|\int_{0}^{t_{l \beta}} C_{p} e^{A_{p}(t-\tau)} B_{p} u_{p}(\tau) \mathrm{d} \tau\right|<\varepsilon_{\beta 2} \\
\forall t>t_{\beta 3} .
\end{array}
$$

Let

$$
t_{u \beta} \geq t_{\beta 3}
$$

Then

$$
\left|\int_{0}^{t_{l \beta}} C_{p} e^{A_{p}\left(t_{u \beta}-\tau\right)} B_{p} u_{p}(\tau) \mathrm{d} \tau\right|<\varepsilon_{\beta 2}
$$


The second term in the RHS of (13) will now be taken up.

$$
\begin{aligned}
& \int_{t_{l \beta}}^{t} C_{p} e^{A_{p}(t-\tau)} B_{p} u_{p}(\tau) \mathrm{d} \tau= {\left[u_{p}(\tau) \int_{t_{l \beta}}^{\tau} C_{p} e^{A_{p}(t-\bar{\tau})} B_{p} \mathrm{~d} \bar{\tau}\right]_{\tau=t_{l \beta}}^{t} } \\
&-\int_{t_{l \beta}}^{t}\left[\int_{t_{l \beta}}^{\tau} C_{p} e^{A_{p}(t-\bar{\tau})} B_{p} \mathrm{~d} \bar{\tau}\right] \dot{u}_{p}(\tau) \mathrm{d} \tau \\
&=\left[u_{p}(t) \int_{t_{l \beta}}^{t} C_{p} e^{A_{p}(t-\bar{\tau})} B_{p} \mathrm{~d} \bar{\tau}\right] \\
&+\int_{t_{l \beta}}^{t} C_{p} e^{A_{p}(t-\tau)} A_{p}^{-1} B_{p} \dot{u}_{p}(\tau) \mathrm{d} \tau \\
&-\int_{t_{l \beta}}^{t} C_{p} e^{A_{p}\left(t-t_{\beta}\right)} A_{p}^{-1} B_{p} \dot{u}_{p}(\tau) \mathrm{d} \tau
\end{aligned}
$$

Consider the first term in the RHS of (16).

$$
\begin{aligned}
u_{p}(t) \int_{t_{l \beta}}^{t} C_{p} e^{A_{p}(t-\bar{\tau})} B_{p} \mathrm{~d} \bar{\tau}= & u_{p}(t)\left[C_{p} e^{A_{p}(t-\bar{\tau})}\left(-A_{p}^{-1}\right) B_{p}\right]_{t_{l \beta}}^{t} \\
= & u_{p}(t)\left[-C_{p} A_{p}^{-1} B_{p}\right] \\
& +u_{p}(t) C_{p} e^{A_{p}\left(t-t_{l \beta}\right)} A_{p}^{-1} B_{p} .
\end{aligned}
$$

As $\lim _{t \rightarrow \infty} C_{p} e^{A_{p}\left(t-t_{\beta}\right)} A_{p}^{-1} B_{p}=0$ and as $u_{p}$ is bounded,

$$
\text { for any } \varepsilon_{\beta 3}>0, \exists t_{\beta 4} \in \Re^{+} \ni\left|u_{p}(t) C_{p} e^{A_{p}\left(t-t_{\beta}\right)} A_{p}^{-1} B_{p}\right|<\varepsilon_{\beta 3} \forall t>t_{\beta 4} \text {. }
$$

Set

$$
t_{u \beta} \geq t_{\beta 4}
$$

Then,

$$
\left|u_{p}\left(t_{u \beta}\right) C_{p} e^{A_{p}\left(t_{u \beta}-t_{l \beta}\right)} A_{p}^{-1} B_{p}\right|<\varepsilon_{\beta 3} .
$$

Hence using $G_{p}(0)=-C_{p} A_{p}^{-1} B_{p}$, it follows from (17) that

$$
\begin{aligned}
{\left[u_{p}\left(t_{u \beta}\right) G_{p}(0)-\varepsilon_{\beta 3}\right] } & <u_{p}\left(t_{u \beta}\right) \int_{t_{l \beta}}^{t_{u \beta}} C_{p} e^{A_{p}\left(t_{u \beta}-\bar{\tau}\right)} B_{p} \mathrm{~d} \bar{\tau} \\
& <\left[u_{p}\left(t_{u \beta}\right) G_{p}(0)+\varepsilon_{\beta 3}\right] .
\end{aligned}
$$

Likewise the second and the final terms in the RHS of (16) can be analysed to yield respectively the following:

$$
\text { for any } \varepsilon_{\beta 4}>0,\left|\int_{t_{l \beta}}^{t_{u \beta}} C_{p} e^{A_{p}\left(t_{u \beta}-\tau\right)} A_{p}^{-1} B_{p} \dot{u}_{p}(\tau) \mathrm{d} \tau\right|<\varepsilon_{\beta 4}
$$


and

$$
\text { for any } \varepsilon_{\beta 5}>0,\left|\int_{t_{l \beta}}^{t_{u \beta}} C_{p} e^{A_{p}\left(t_{u \beta}-t_{l \beta}\right)} A_{p}^{-1} B_{p} \dot{u}_{p}(\tau) \mathrm{d} \tau\right|<\varepsilon_{\beta 5} \text {. }
$$

Using (20), (21) and (22) in (16) and rearranging,

$$
\begin{aligned}
u_{p}\left(t_{u \beta}\right) G_{p}(0)-\left[\varepsilon_{\beta 3}+\varepsilon_{\beta 4}+\varepsilon_{\beta 5}\right] & <\int_{t_{l \beta}}^{t_{u \beta}} C_{p} e^{A_{p}\left(t_{u \beta}-\tau\right)} B_{p} u_{p}(\tau) \mathrm{d} \tau \\
& <u_{p}\left(t_{u \beta}\right) G_{p}(0)+\left[\varepsilon_{\beta 3}+\varepsilon_{\beta 4}+\varepsilon_{\beta 5}\right] .
\end{aligned}
$$

Substituting (23) and (15) into (13) and simplifying,

$$
\begin{aligned}
u_{p}\left(t_{u \beta}\right) G_{p}(0)-\left[\varepsilon_{\beta 2}+\varepsilon_{\beta 3}+\varepsilon_{\beta 4}+\varepsilon_{\beta 5}\right] & <y_{p}\left(t_{u \beta}\right)<u_{p}\left(t_{u \beta}\right) G_{p}(0) \\
& +\left[\varepsilon_{\beta 2}+\varepsilon_{\beta 3}+\varepsilon_{\beta 4}+\varepsilon_{\beta 5}\right]
\end{aligned}
$$

$G_{p}(0) \neq 0$ by (A5).

Inequality (24) was derived with the initial assumption (6), i.e., $\beta(t)=1, t \in\left[t_{l \beta}, t_{u \beta}\right]$. A scrutiny of the derivation is, however, sufficient to reveal that even if $\beta(t)=-1, t \in$ $\left[t_{l \beta}, t_{u \beta}\right]$, (24) would hold.

Before proceeding further with (24), it shall be shown that when $t_{u \beta}$ exceeds a threshold value, $u_{p}\left(t_{u \beta}\right)$ must necessarily be non-zero regardless of whether $\beta(t)=1$ or -1 .

Since $\lim _{t \rightarrow \infty} u_{m}(t)=M_{m} \neq 0$,

$$
\begin{aligned}
& \text { for any } \varepsilon_{\beta 6} \in\left(0,\left|M_{m}\right|\right) \text {, } \\
& \qquad \exists t_{\beta 5} \in \mathfrak{R}^{+} \ni M_{m}-\varepsilon_{\beta 6}<u_{m}(t)<M_{m}+\varepsilon_{\beta 6} \forall t \geq t_{\beta 5}
\end{aligned}
$$

Let

$$
t_{u \beta} \geq t_{\beta 5}
$$

Then as $\alpha>0, u_{p}\left(t_{u \beta}\right)=\beta\left(t_{u \beta}\right) \alpha\left(t_{u \beta}\right) u_{m}\left(t_{u \beta}\right) \neq 0$. Consequently $\varepsilon_{\beta 2}, \varepsilon_{\beta 3}, \varepsilon_{\beta 4}$ and $\varepsilon_{\beta 5}$, which are arbitrarily small, can be so chosen that

$$
0<\left[\varepsilon_{\beta 2}+\varepsilon_{\beta 3}+\varepsilon_{\beta 4}+\varepsilon_{\beta 5}\right] \ll\left|u_{p}\left(t_{u \beta}\right)\right| .\left|G_{p}(0)\right| .
$$

When (24) is seen in the light of (26), it is apparent that $y_{p}\left(t_{u \beta}\right) \neq 0$, that

$$
\operatorname{sgn}\left\{y_{p}\left(t_{u \beta}\right)\right\}=\operatorname{sgn}\left\{u_{p}\left(t_{u \beta}\right) G_{p}(0)\right\}
$$

and that

$$
\operatorname{sgn}\left\{u_{p}\left(t_{u \beta}\right) y_{p}\left(t_{u \beta}\right)\right\}=\operatorname{sgn}\left\{u_{p}\left(t_{u \beta}\right)\right\} \operatorname{sgn}\left\{u_{p}\left(t_{u \beta}\right) G_{p}(0)\right\}=\operatorname{sgn}\left\{G_{p}(0)\right\}
$$

Constraints (8) and (11) have to be simultaneously satisfied by $t_{l \beta}$. Let

$$
t_{l \beta} \geq \max \left\{t_{\beta 1}, t_{\beta 2}\right\}
$$


Constraints (14), (18) and (25) have to be simultaneously satisfied by $t_{u \beta}$ which, in addition, has to exceed $t_{l \beta}$. Choose

$$
t_{u \beta} \geq \max \left\{t_{l \beta}, t_{\beta 3}, t_{\beta 4}, t_{\beta 5}\right\} .
$$

From (28) and (29), $t_{l \beta}$ and $t_{\mu \beta}$ can be set such that for some $i$, say $j$,

$$
t_{l \beta}>2^{j} T_{\beta} \text { and } t_{l \beta}<t_{u \beta}=2^{j+1} T_{\beta} .
$$

Equation (27) now implies $\operatorname{sgn}\left\{u_{p}\left(2^{j+1} T_{\beta}\right) y_{p}\left(2^{j+1} T_{\beta}\right)\right\}=\operatorname{sgn}\left\{G_{p}(0)\right\}$ and thence,

$$
\operatorname{sgn}\left\{G_{m}(0) u_{p}\left(2^{j+1} T_{\beta}\right) y_{p}\left(2^{j+1} T_{\beta}\right)\right\}=\operatorname{sgn}\left\{G_{m}(0) G_{p}(0)\right\} .
$$

It is emphasized here that (30) is true independent of the assumption that (7) holds for all $t>t_{l \beta}$. Also, it has been seen that $\beta(t)=-1$ for $t \in\left[t_{l \beta}, t_{u \beta}\right]$ leads to the same result.

When (30) is compared with the definition of $\beta$ it is clear that regardless of what $\beta(t)$ for $t \in\left(2^{j} T_{\beta}, 2^{j+1} T_{\beta}\right]$ was,

$$
\beta(t)=\operatorname{sgn}\left\{G_{m}(0) G_{p}(0)\right\}, t \in\left(2^{j+1} T_{\beta}, 2^{j+2} T_{\beta}\right] .
$$

Hence it is established that

$$
\lim _{t \rightarrow \infty} \beta(t)=\operatorname{sgn}\left\{G_{m}(0) G_{p}(0)\right\} .
$$

Evaluation of $\lim _{t \rightarrow \infty}\left|u_{p}(t)\right|$ and $\lim _{t \rightarrow \infty}\left|y_{p}(t)\right|$. Let $\alpha_{f} \triangleq \lim _{t \rightarrow \infty} \alpha(t)$. We can arrive at

$$
\alpha_{f}=\left\lfloor\left[\left|\frac{G_{m}(0)}{G_{p}(0)}\right|^{\frac{1}{2}}\right] \mid\right.
$$

Then

$$
\lim _{t \rightarrow \infty}\left|u_{p}(t)\right|=\left\lfloor\left[\left|\frac{G_{m}(0)}{G_{p}(0)}\right|^{\frac{1}{2}}\right]|\cdot| M_{m} \mid\right.
$$

Hence $\lim _{t \rightarrow \infty}\left|y_{p}(t)\right|=\left|\left\{\left|G_{m}(0) G_{p}(0)\right|^{\frac{1}{2}}\right\}\right| .\left|M_{m}\right|$.

This completes the proof of parts 2 and 3 .

The role of $\beta$ and evaluation of $J_{f}$. Applying lemma 1 to the plant excited by $u_{p}$, we get

$$
\lim _{t \rightarrow \infty} \frac{1}{t} \int_{0}^{t} y_{p}^{2}(\tau) \mathrm{d} \tau=\left|G_{m}(0) G_{p}(0)\right| M_{m}^{2}
$$

Now,

$$
\lim _{t \rightarrow \infty} u_{p}(t)=\lim _{t \rightarrow \infty} \alpha(t) \beta(t) u_{m}(t)=\left|\left\{\left|\frac{G_{m}(0)}{G_{p}(0)}\right|^{\frac{1}{2}}\right\}\right| M_{m}\left\{\lim _{t \rightarrow \infty} \beta(t)\right\}
$$


Table 1. Role of $\beta$.

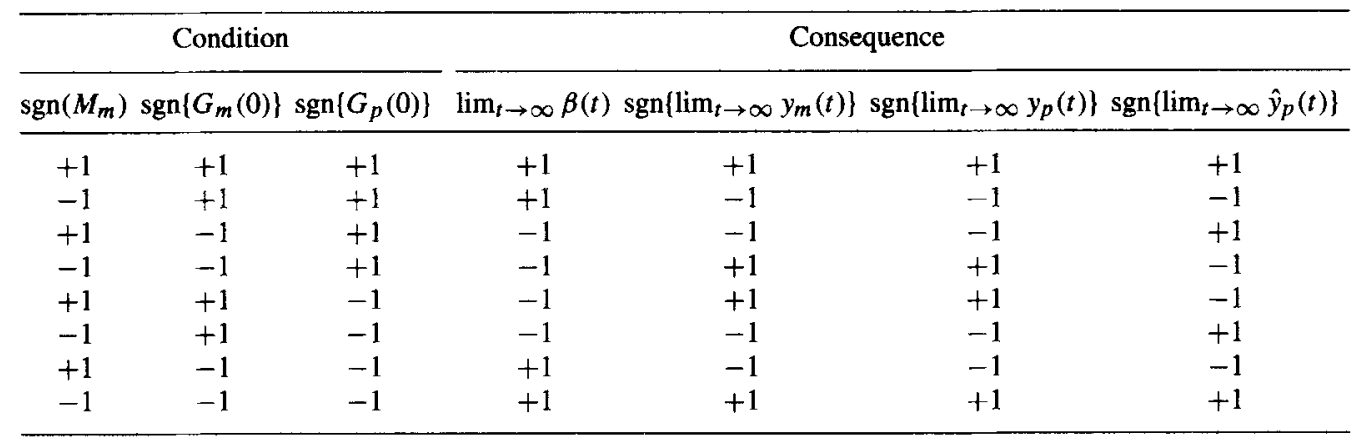

Note. Here $\hat{y}_{p}(t)$ denotes the plant output if $\beta \equiv 1$.

and thence,

$$
\lim _{t \rightarrow \infty} y_{p}(t)=G_{p}(0)\left\{\lim _{t \rightarrow \infty} u_{p}(t)\right\}=G_{p}(0)\left|\left\{\left|\frac{G_{m}(0)}{G_{p}(0)}\right|^{\frac{1}{2}}\right\}\right| M_{m}\left\{\lim _{t \rightarrow \infty} \beta(t)\right\}
$$

We have seen that

$$
\lim _{t \rightarrow \infty} y_{m}(t)=G_{m}(0) M_{m} .
$$

Table 1 brings out the effectiveness of $\beta$ in ensuring that $\lim _{t \rightarrow \infty} y_{p}(t)$ has the same sign as $\lim _{t \rightarrow \infty} y_{m}(t)$. In developing the table, (32), (34) and (35) have been used. All permissible combinations of $\operatorname{sgn}\left\{M_{m}\right\}, \operatorname{sgn}\left\{G_{m}(0)\right\}$ and $\operatorname{sgn}\left\{G_{p}(0)\right\}$ are considered.

It can be seen that for all combinations of $M_{m}, G_{m}(0)$ and $G_{p}(0)$, the model and the plant outputs are of the same sign as $t \rightarrow \infty$. On the other hand, if $\beta \equiv 1$, i.e., if the control law of corollary 1 were employed, the model and plant outputs would have different signs, as $t \rightarrow \infty$, whenever $G_{m}(0)$ and $G_{p}(0)$ have different signs.

It is now straightforward to show that

$$
\lim _{t \rightarrow \infty} \frac{1}{t} \int_{0}^{t} y_{m}(\tau) y_{p}(\tau) \mathrm{d} \tau=G_{m}(0) G_{p}(0)\left|\left\{\left|\frac{G_{m}(0)}{G_{p}(0)}\right|^{\frac{1}{2}}\right\}\right| M_{m}^{2}\left\{\lim _{t \rightarrow \infty} \beta(t)\right\}
$$

Using (4), (33) and (36) and simplifying using table 1, we get

$$
\begin{aligned}
& \lim _{t \rightarrow \infty} \frac{1}{t} \int_{0}^{t}\left\{y_{m}(\tau)-y_{p}(\tau)\right\}^{2} \mathrm{~d} \tau \\
& \quad=G_{m}^{2}(0) M_{m}^{2}+\left|G_{m}(0) G_{p}(0)\right| M_{m}^{2}-2\left|\left\{\left|G_{m}(0)\right|^{\frac{3}{2}}\left|G_{p}(0)\right|^{\frac{1}{2}}\right\}\right| M_{m}^{2}
\end{aligned}
$$

Now by (37) and (4),

$$
d\left(u_{m}\right)=\left[G_{m}^{2}(0)+\left|G_{m}(0) G_{p}(0)\right|-2\left|\left\{\left|G_{m}(0)\right|^{\frac{3}{2}}\left|G_{p}(0)\right|^{\frac{1}{2}}\right\}\right|\right] / G_{m}^{2}(0) .
$$


We make here an interesting observation, namely, $d\left(u_{m}\right)$ is independent of $M_{m}$. Hence the RHS of (38) remains the same for all $u_{m} \in \Omega_{u, f}$. Therefore

$$
J_{f}=\left[G_{m}^{2}(0)+\left|G_{m}(0) G_{p}(0)\right|-2\left|\left\{\left|G_{m}(0)\right|^{\frac{3}{2}}\left|G_{p}(0)\right|^{\frac{1}{2}}\right\}\right|\right] / G_{m}^{2}(0) .
$$

We now provide an alternative sign-matching scheme in the law of lemma 4 to follow. In view of lemma 3 and its proof, the motivation behind this scheme is relatively easy to grasp. We shall confine ourselves to making a few cursory comments on the similarities and differences between the two sign-matching schemes.

Lemma 4. Lemma 3 holds even if $\beta$ there is replaced by

$$
\beta(t) \triangleq\left\{\begin{aligned}
1 ; & 0 \leq t \leq T_{1}+\varepsilon, \varepsilon \in \mathfrak{R}^{+} \\
-1 ; & t>T_{1}+\varepsilon, G_{m}(0)[1 /(t+\varepsilon)] \int_{0}^{t-\varepsilon} u_{p}(\tau) y_{p}(\tau) \mathrm{d} \tau<0 \\
1 ; & \text { else. }
\end{aligned}\right.
$$

The underlying principle that is exploited in both sign-matching schemes is essentially the same. In fact, table 1 is valid here also. The primary variation is that $\beta$ here is adapted continuously instead of at pre-specified instants as before. As a result, the computational effort involved here is relatively more. The time at which $\beta$ settles to its final value indeed depends on the choice of $T_{\beta}$.

Lemma 3 demonstrates how the introduction of $\beta$ in the control law ensures the desired sign-matching. Thus both minimum and non-minimum phase plants and reference models have been successfully taken care of. In this sense, the control law of lemma 3 is a refinement of the law of corollary 1. It is, nonetheless, not optimal as judged by the performance index. Both these aspects are brought out in example 2.

Example 2.

$$
\begin{aligned}
G_{m}(s) & =\frac{8(s+3)}{(s+2)(s+4)} ; \\
G_{p}(s) & =\frac{(s-9)}{(s+1)(s+10)} ; \quad u_{m}(\cdot) \in \Omega_{u, f} ; \quad M_{m}=1 .
\end{aligned}
$$

With the law of corollary $1, u_{p}=\alpha u_{m}$, the $y_{m}(t) \rightarrow 3.0, \alpha_{f}=1.8257, y_{p}(t) \rightarrow$ -1.6432 and $J_{f}=2.3954$. This large value of $J_{f}$ is obtained because as $t \rightarrow \infty, y_{m}(t)$ and $y_{p}(t)$ have opposite signs even though their magnitudes are close. Else, if the control law of lemma $3, u_{p}=\alpha \beta u_{m}$, were employed then $y_{p}(t) \rightarrow 1.6432$ due to $\beta(t)$ and $J_{f}=0.2046$. Thus lemma 3 is a refinement of corollary 1 .

Now, choose $u_{p}=-y_{m}$. Then $J_{f}=0.01$. Thus the scheme of lemma 3 is sub-optimal.

Obviously, the control law, $u_{p}=-y_{m}$, can perform miserably for some other $G_{p}(s)$, whereas $u_{p}=\alpha \beta u_{m}$ will continue to force $J_{f}$ to the value specified in lemma 3 . The former control was chosen having the details $G_{m}(s)$ and $G_{p}(s)$ in mind. As $G_{p}(s)$ is indeed unknown, $J_{f}$ cannot be computed $a$ priori. Nonetheless, lemma 3 specifies exactly how $J_{f}$ depends on $G_{m}(s)$ and $G_{p}(s)$.

With this, corollary 1 and lemma 3 (or lemma 4) set the stage for the optimal control schemes that follow. 


\section{On-line-adaptive-optimal schemes}

The schemes of $\S 3$ do not ensure the desired asymptotic matching of the model output by the plant output. It was remarked (vide remark 3 ) that $\lim _{t \rightarrow \infty} \alpha(t)=1$ is necessary and, further, that such an $\alpha$ is dispensable from the control law in the context of corollary 1 . This holds even in respect of the laws of lemmas $3 \& 4$. Naturally, the question that we seek to answer is the following: Which bounded function, say $\gamma$, of $\alpha$, simultaneously ensures, in the present framework, the convergence $\alpha \rightarrow 1$ and, in turn, itself converges to $\left|G_{m}(0) / G_{p}(0)\right|$ ? If we succeed in constructing such a function, we would have solved the problem posed in $\S 2$. This is precisely the motivation for the gain-matching schemes. Signmatching as ensured by lemmas 3 or 4 must indeed continue to hold. A scheme $\mathcal{A}_{f}$ which proposes a $\gamma(\alpha)$ which is updated only at specified instants of time, and which is shown to be $\varepsilon$-optimal, has been reported (Shankar 1993). Algorithm $\mathcal{A}_{f o}$ to be proposed here is in pursuit of optimality. Unlike in $\mathcal{A}_{f}$, adaptation in $\mathcal{A}_{f o}$ is continuous. A second optimal scheme, $\mathcal{A}_{f o}^{*}$, that is capable of 'improving', in some sense, on $\mathcal{A}_{f o}$ will then be presented. Finally, corollary 2, below, shows how the presence of non-zero initial conditions of the plant is not a deterrent to the results presented in this paper.

Algorithm $\mathcal{A}_{f o}$ : The system is setup as in figure 1.

$u_{m}(\cdot) \in \Omega_{u, f}, \alpha(\cdot)$, as given in lemma 3, and

$\beta(\cdot)$, as given in lemmas 3 or 4 .

$$
\gamma(t) \triangleq\left\{\begin{array}{l}
1 ; 0 \leq t \leq T_{1}+\varepsilon, \varepsilon \in \mathfrak{R}^{+} \\
1+\eta \int_{0}^{t}-\varepsilon\left\{\frac{\alpha(\tau)-1}{\alpha(\tau)+1}\right\} \mathrm{d} \tau \\
\quad t>T_{1}+\varepsilon,\left\{1+\eta \int_{0}^{t}-\varepsilon\left\{\frac{\alpha(\tau)-1}{\alpha(\tau)+1}\right\} \mathrm{d} \tau \geq 0\right\}, \eta \in \mathfrak{R}^{+} \\
0 ; \text { else, }
\end{array}\right.
$$

The control law: $u_{p}(\cdot) \triangleq \beta(\cdot) \gamma(\cdot) u_{m}(\cdot)$.

Theorem 1. Let the control law be given by algorithm $\mathcal{A}_{f o}$. Then

(1) $\alpha, \gamma, u_{p}$ and $y_{p}$ are bounded;

(2) $\lim _{t \rightarrow \infty} \alpha(t)=1$;

(3) $\lim _{t \rightarrow \infty} \beta(t)=\operatorname{sgn}\left\{G_{m}(0) G_{p}(0)\right\}$;

(4) $\lim _{t \rightarrow \infty} \gamma(t)=\left|G_{m}(0) / G_{p}(0)\right|$;

(5) $\lim _{t \rightarrow \infty} u_{p}(t)=\operatorname{sgn}\left\{G_{m}(0) G_{p}(0)\right\}\left|G_{m}(0) / G_{p}(0)\right| M_{m}$;

(6) $\lim _{t \rightarrow \infty} y_{p}(t)=\lim _{t \rightarrow \infty} y_{m}(t)=G_{m}(0) M_{m}$; and

(7) the performance index $J_{f}=0$. 
Outline of proof. It may be observed that by definition, $\gamma \geq 0$. The existence of $\alpha_{f} \triangleq$ $\lim _{t \rightarrow \infty} \alpha(t)$ is ensured by proposition 2 as before.

$$
\left\{\alpha_{f}<1\right\} \Rightarrow\left\{\lim _{t \rightarrow \infty} \gamma(t)=0\right\} \Rightarrow\left\{\lim _{t \rightarrow \infty} y_{p}(t)=0\right\} \Rightarrow\left\{\alpha_{f}=\infty\right\}
$$

a contradiction. It can also be shown that

$$
\left\{\alpha_{f}>1\right\} \Rightarrow\left\{\lim _{t \rightarrow \infty} \gamma(t)=\infty\right\} \Rightarrow\left\{\lim _{t \rightarrow \infty}\left|y_{p}(t)\right|=\infty\right\} \Rightarrow\left\{\alpha_{f}=0\right\}
$$

a contradiction again. Thus $\alpha_{f}=1$.

Note that for $\lim _{t \rightarrow \infty} \gamma(t)$ to exist, it is sufficient that the integrand $[(\alpha-1) /(\alpha+1)] \rightarrow$ 0 . This in turn can happen iff $\alpha_{f}=1$. So $\lim _{t \rightarrow \infty} \gamma(t)$ exists.

Hereafter, after ensuring the existence $\lim _{t \rightarrow \infty} \beta(t)$ as before, the various limits can be directly evaluated and the rest of the theorem proved.

The problem stated in $\S 2$ is hereby solved.

We are now in a position to propose an alternative optimal scheme, $\mathcal{A}_{f o}^{*}$. The cue for this second scheme is contained in the fact that by theorem $1, \alpha_{f}=1$.

Algorithm $\mathcal{A}_{f o}^{*}$. This algorithm is identical to algorithm $\mathcal{A}_{f o}$ except that the control law is given by

$$
u_{p}(\cdot) \triangleq \alpha(\cdot) \beta(\cdot) \gamma(\cdot) u_{m}(\cdot)
$$

Theorem 2. The results of theorem 1 hold with the control law specified by algorithm $\mathcal{A}_{f o}^{*}$.

The proof readily follows from the proof of theorem 1 taken with the results that $\alpha$ is bounded and it smoothly settles at unity. After all, in that event, the control of $\mathcal{A}_{\text {fo }}^{*}$ approaches the control of $\mathcal{A}_{f o}$.

$\mathcal{A}_{f o}^{*}$ is optimal like $\mathcal{A}_{f o}$ in the sense of $J_{f}$. Also, in both the cases, to start with, $\alpha(t)=1, t \in\left[0, T_{1}\right]$, and finally, $\alpha_{f}=1$. It may therefore appear on the face of it that $\mathcal{A}_{\text {fo }}^{*}$ will contribute a mere unnecessary transient excursion. That, however, is not the case. It does possess some additional interesting properties. We will reserve our comments on this issue until after presenting the simulations of the two laws.

Non-zero initial conditions: In all the schemes discussed thus far, zero initial conditions were assumed (vide $\S 3$ ); i.e., $x_{p}\left(0_{+}\right)=0$. As the plant considered here is asymptotically stable, any unforced dynamics (due to non-zero initial conditions) has to decay smoothly and at an exponential rate. Such dynamics, if any, may be looked upon as the effect of some specific, yet unknown, finite-time perturbation in the control. Seen in this light, the results presented in this paper are not compromised. This aspect is formally brought out in the following corollary.

\section{COROLLARY 2}

Corollary 1, lemmas 3 and 4 , and theorems 1 and 2 hold even if $\alpha(\cdot)$ is consistently replaced by $\tilde{\alpha}(\cdot)$ where 


$$
\tilde{\alpha}(t) \triangleq\left\{\begin{array}{cc}
p(t) ; \quad 0 \leq t \leq T_{1}+\varepsilon, T_{1}, \varepsilon \in \mathfrak{R}^{+}, \\
\left.\quad \exists t_{1} \in\left(0, T_{1}\right) \ni y_{m}\left(t_{1}\right) y_{p}\left(t_{1}\right) \neq 0\right\} \\
\alpha(t) ; \quad \text { else } ;
\end{array}\right.
$$

and $p:\left[0, T_{1}\right] \longmapsto \Re$ is bounded and continuous or has utmost finitely many discontinuities of the first kind.

It may be noted that the condition

$$
\left\{\exists t_{1} \in\left(0, T_{1}\right) \ni y_{m}\left(t_{1}\right) y_{p}\left(t_{1}\right) \neq 0\right\}
$$

sets only a lower bound for $T_{1} . T_{1}$ is simply required to be greater than the time at which $u_{m}$ begins to activate the outputs of the model and the plant.

Remark 5. Before closing this section a few words on the formulation of the various schemes proposed in this paper will be in order. With an eye on tracking plants that exhibit. either continuous/frequent, small drifts or relatively large, infrequent jumps, the schemes are designed to be continually sensitive to any deviation from the desired performance and to modify the control suitably. This puts the schemes proposed as also the proofs given here in the proper perspective.

\section{Simulations}

Reporting simulations here serves a dyad of purposes. First, to facilitate comparison between the control laws presented in this paper so as to demonstrate the progressive refinement as remarked in the previous sections. Second, to provide an insight into the roles played by $T_{1}$ and $\eta$ which are to be chosen by the user, i.e., to highlight how, using the same law, better transient responses may be obtained by suitable choice of $T_{1}$ and $\eta$. In all simulations, therefore, we use the same reference model, $G_{m}(s)$, and one of the two plants, $G_{p 1}(s)$ and $G_{p 2}(s)$. Thus,

$$
\begin{aligned}
& \text { reference model }: G_{m}(s)=1 /(s+2), \\
& \begin{aligned}
\text { plant } 1: G_{p 1}(s) & =(s+2) /[(s+1)(s+3)(s+4)] \text { and } \\
\text { plant } 2: G_{p 2}(s) & =(s-2) /[(s+1)(s+3)(s+4)] .
\end{aligned}
\end{aligned}
$$

Plant 1 is minimum phase like the model, whereas plant 2 is nonminimum phase. They are both chosen to have the same poles to bring to focus the effect of the change in the location of the zero. It may be observed that the model has no pole in common with either of the plants. We have

$$
G_{m}(0) / G_{p 1}(0)=-G_{m}(0) / G_{p 2}(0)=3,
$$

which is the desired magnitude of the controller gain.

In all cases, the reference model input is chosen as $u_{m}(t)=5 u(t)$, where $u(t)$ is the standard unit step. $\varepsilon=0.1$ throughout.

The plant response in the absence of control is designated $y_{p}^{\prime}$ and is shown along with $y_{m}$ and $y_{p}$. 


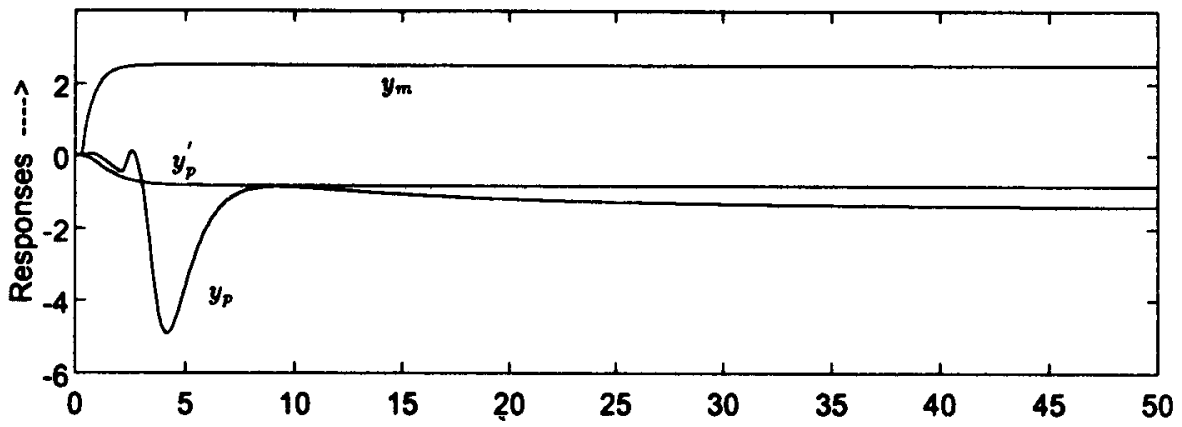

(a)

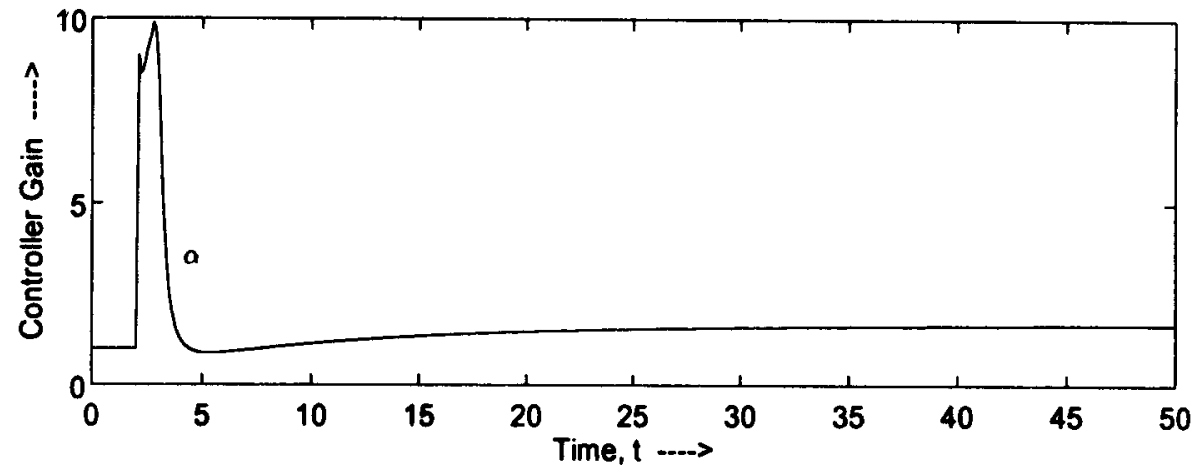

(b)

Figure 2. (a) Reference model and plant responses, and (b) controller performance for case 1.
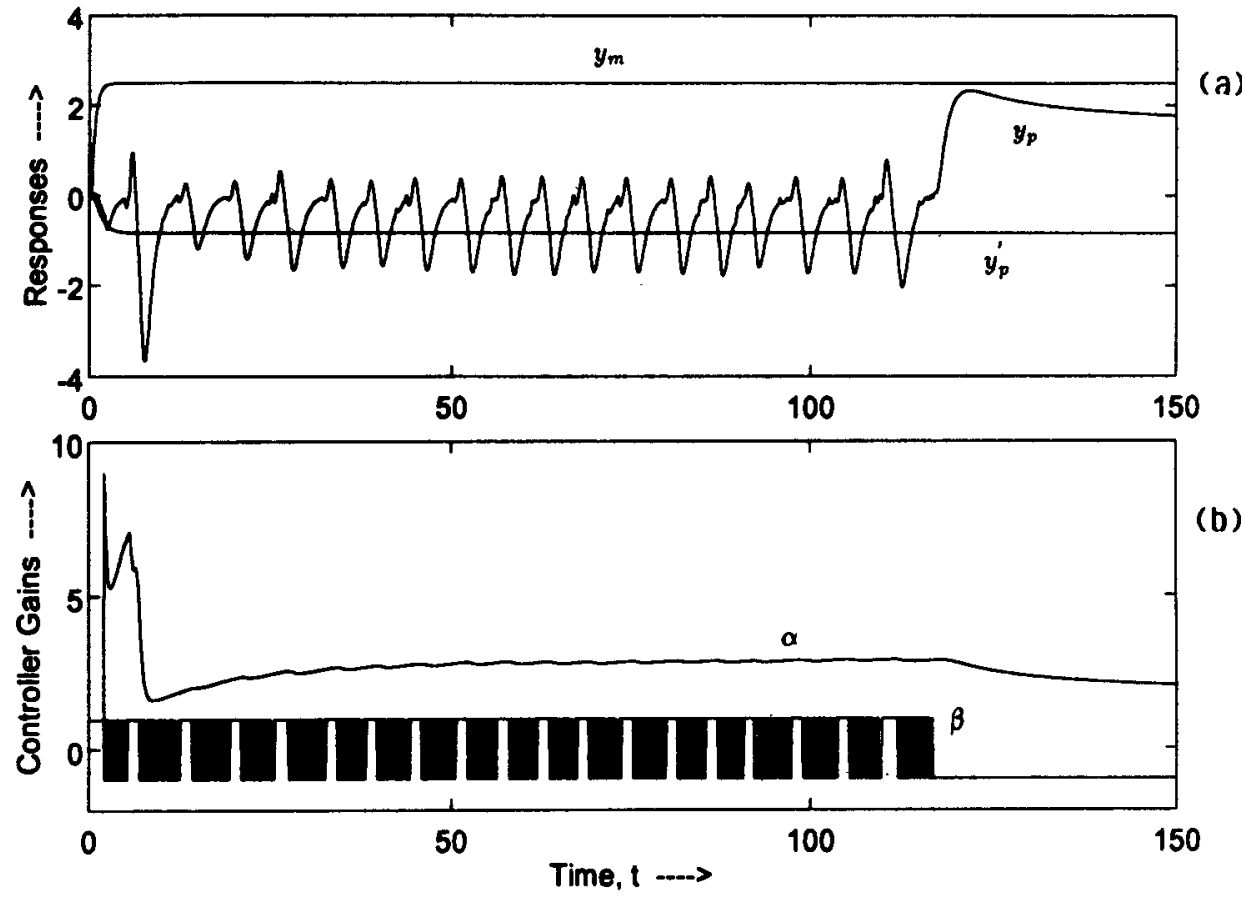

Figure 3. (a) Reference model and plant responses, and (b) controller performance for case 2. 

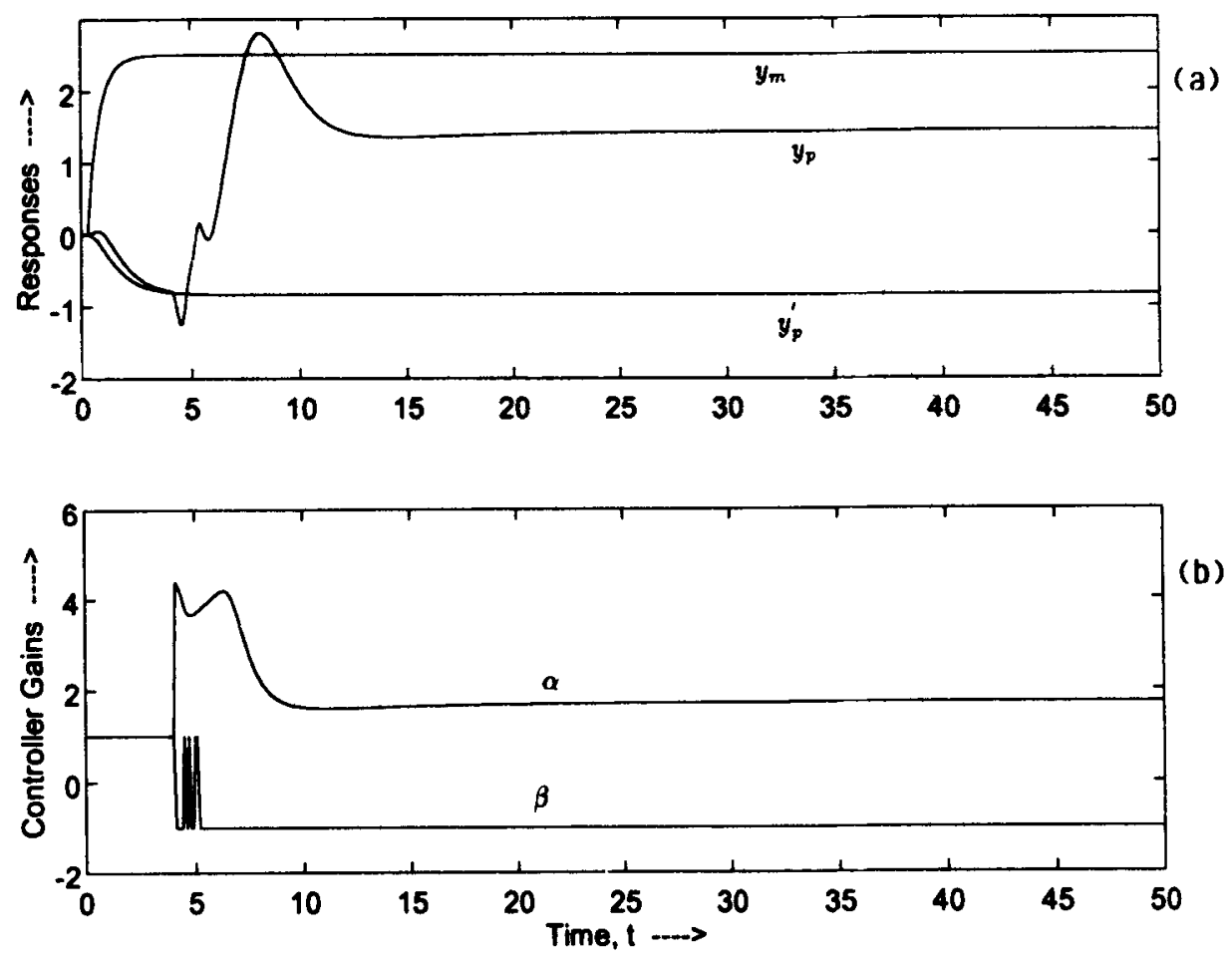

Figure 4. (a) Reference model and plant responses, and (b) controller performance for case 3.

Case 1. Plant 2; the control $u_{p}=\alpha u_{m}$ of lemma $2 ; T_{1}=2.0 ; \eta=0.2 ;$ figure $2 . \alpha \rightarrow \sqrt{3}$ and sign-mismatch exists as expected.

Case 2. Plant 2; the control $u_{p}=\alpha \beta u_{m}$ of lemma $4 ; T_{1}=2.0 ; \eta=0.2 ;$ figure $3 . \alpha \rightarrow \sqrt{3}$ as before but sign-matching is achieved. The prolonged transient in $y_{p}$ is due to the delay in $\beta$ settling at -1 . A better transient response is often desirable.

Case 3 below is meant to show how a different choice of $T_{1}$ in the otherwise same situation can provide a substantially improved $y_{p}$.

Note. The difference in the spreads of the time axes between one figure and another has to be recognized before interpreting the plots.

Case 3. Plant 2; the control, $u_{p}=\alpha \beta u_{m}$ of lemma $4 ; T_{1}=1.0 ; \eta=0.2$; figure 4 . While the asymptotic behaviour is as in case 2 , the following improvements are noteworthy.

(i) Much better transient behaviour of $y_{p}$ and faster convergence.

(ii) Quicker settling of $\beta$ to -1 .

(iii) $\alpha$ not only settles faster, but its peak value is reduced by a factor of 2 .

Case 4. Plant 1; the control $u_{p}=\beta \gamma u_{m}$ of algorithm $\mathcal{A}_{f o}$ with $\beta$ of lemma $4 ; T_{1}=2.0$; $\eta=0.2$; figure 5 . $\alpha \rightarrow 1$ unlike in earlier cases. $\beta$ remains at +1 . The convergence of $\gamma$ to 

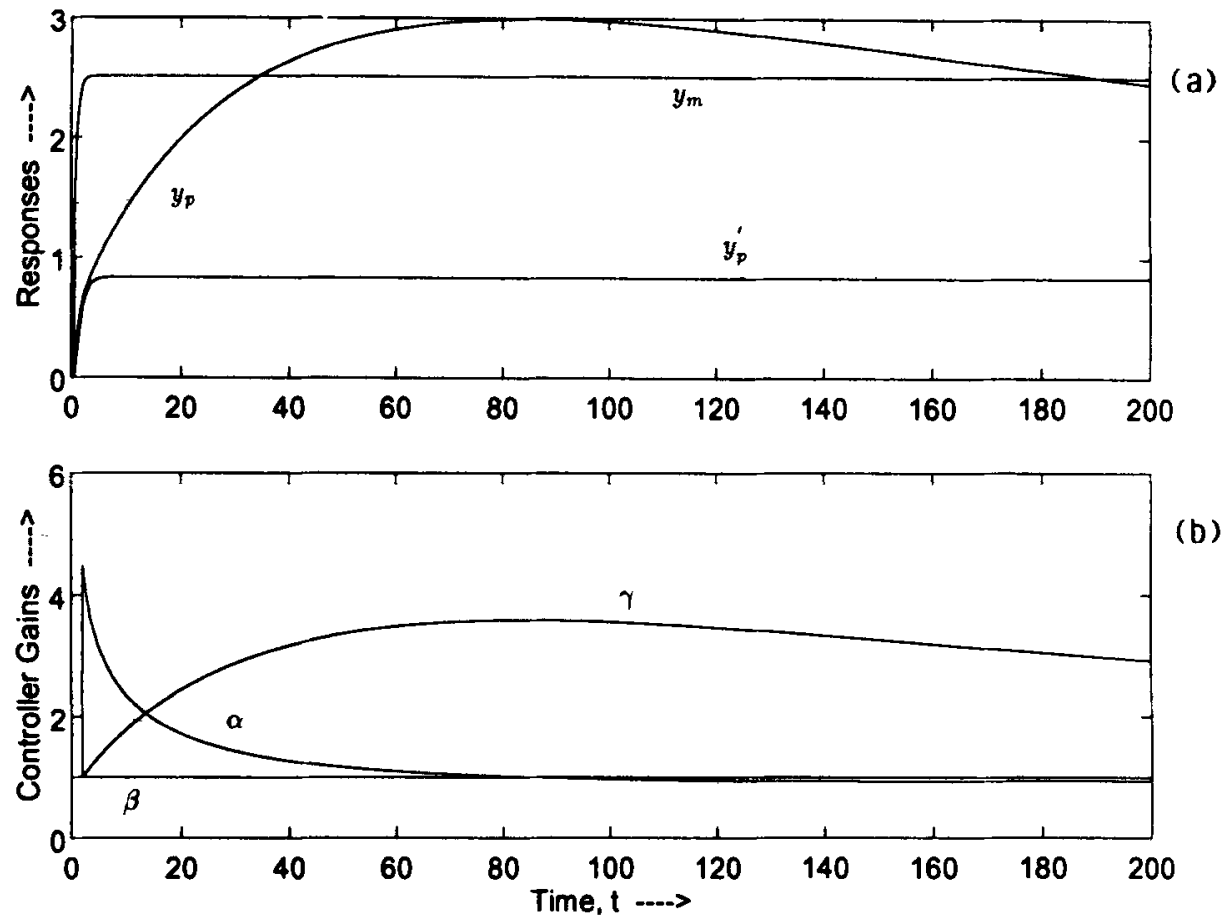

Figure 5. (a) Reference model and plant responses, and (b) controller performance for case 4.
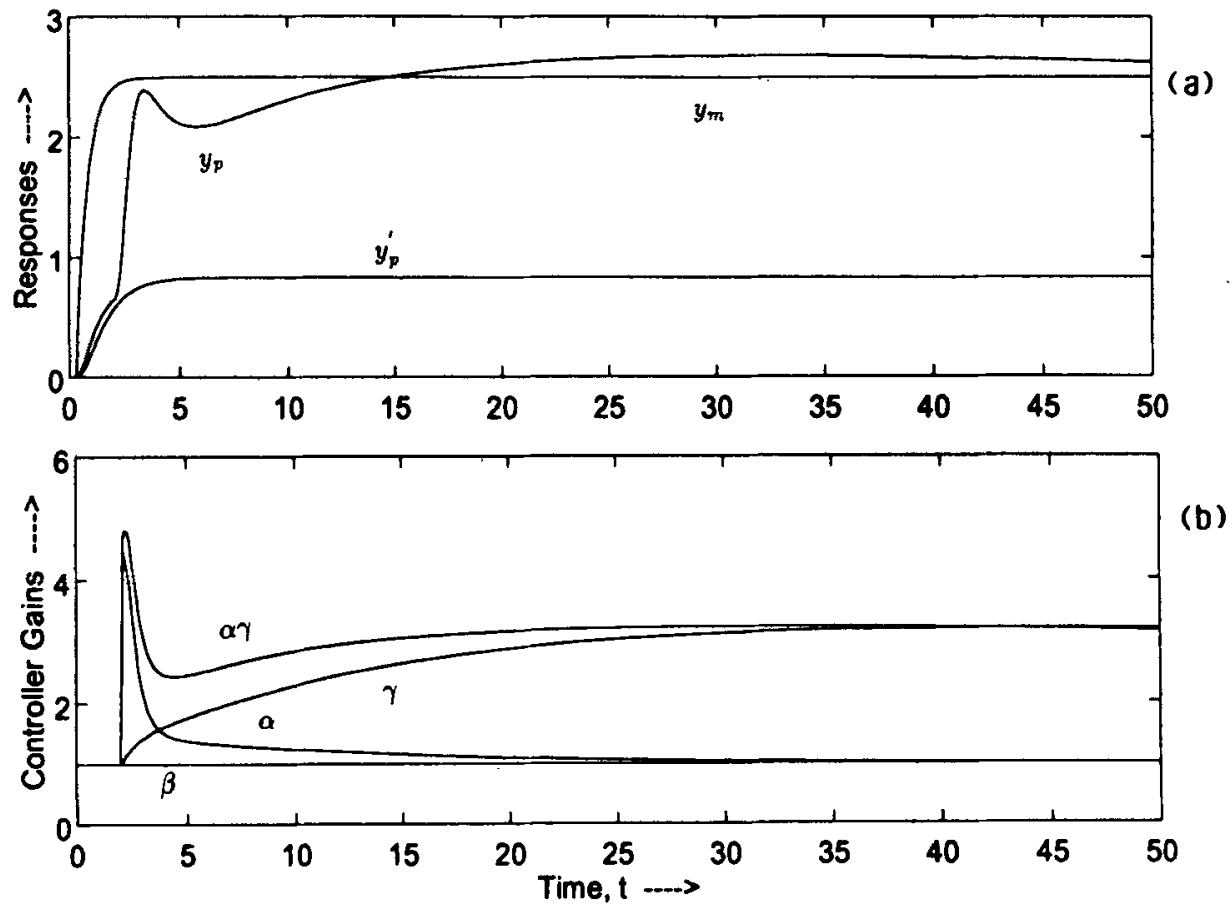

Figure 6. (a) Reference model and plant responses, and (b) controller performance for case 5. 
3 , the desired value, is the highlight in this case. Amplitude-matching is achieved, though not yet so upto $t=200$.

The rate of convergence of $\gamma$, and thence of $y_{p}$, however, leaves much to be desired. It was this desire that motivated, as stated more than once in $\S 4$, the second algorithm $\mathcal{A}_{f o}^{*}$ which, though optimal like $\mathcal{A}_{f o}$ in the sense of $J_{f}$, is an 'improvement' over the latter. Case 5 is meant to demonstrate this fact.

Case 5. Plant 1; the control $u_{p}=\alpha \beta \gamma u_{m}$ of algorithm $\mathcal{A}_{f o}^{*}$ with $\beta$ of lemma 4; $T_{1}=2.0$; $\eta=1.0$; figure 6. Observe that the plots are for $t \in[0,50]$ here. The initial excursion of $\alpha$ from unity has evidently contributed to the improvement in the overall gain $\alpha \gamma$ and hence in $y_{p}$, in addition to reshaping $\gamma$ itself for the better. Of further interest is the faster convergence of $\alpha$ itself in this case as compared to case 4 . Indeed, our claim that algorithm $\mathcal{A}_{f o}^{*}$ compares favourably with algorithm $\mathcal{A}_{f o}$ is amply justified.

Even here, there is scope for fine-tuning the controller by suitably choosing $\eta$. Consider case 6 .

Case 6. Plant 1; the control $u_{p}=\alpha \beta \gamma u_{m}$ of algorithm $\mathcal{A}_{f o}^{*}$ with $\beta$ of lemma 4; $T_{1}=2.0$; $\eta=2.0$; figure 7. $\alpha, \gamma, \alpha \gamma$ and $y_{p}$ - each of these is seen to be more desirable here in comparison to case 5 .

Case 7. Plant 2; the control $u_{p}=\alpha \beta \gamma u_{m}$ of algorithm $\mathcal{A}_{f o}^{*}$ with $\beta$ of lemma $4 ; T_{1}=2.0$; $\eta=0.2$; figure 8 . $\beta$ comes into play here. The trajectories of the gains and of $y_{p}$ before and after convergence of $\beta$ to -1 are markedly different. The efficacy of the algorithm $\mathcal{A}_{f o}^{*}$ is borne out clearly.

Finally, we seek to answer the following question: With any of the schemes proposed in this paper, for plant 2 (which is, of course, unknown), can we improve upon the response of case 7? The last case to follow provides an answer. Only one change is made from case 7, i.e., $T_{1}$ is set to 0.5 .

Case 8. Plant 2; the control $u_{p}=\alpha \beta \gamma u_{m}$ of algorithm $\mathcal{A}_{f o}^{*}$ with $\beta$ of lemma $4 ; T_{1}=0.5$; $\eta=0.2$; figure 9. The answer is evident, indeed. While the peak value of the overall gain $\alpha \gamma$ may have gone up compared to that in case 7 , it has, paradoxically, contributed to a decrease in the peak overshoot of $y_{p}$.

Remark 6. A systematic analysis of the simulations reveals an interesting phenomenon. The successive refinements in the laws is due to the improvements in the individual factors involved in the control. When even one of $\alpha, \beta, \gamma$ or $y_{p}$ moves towards its desirable value, it induces all the others toward their respective desirable values. Conversely, any tendency to undesirable behaviour on the part of any one of the above variables reflects in all the rest so that such a tendency is effectively countered. This combination of inverse relationships between some signals and boot-strapping within the same signal is seen to yield a significant enhancement in the overall performance. 

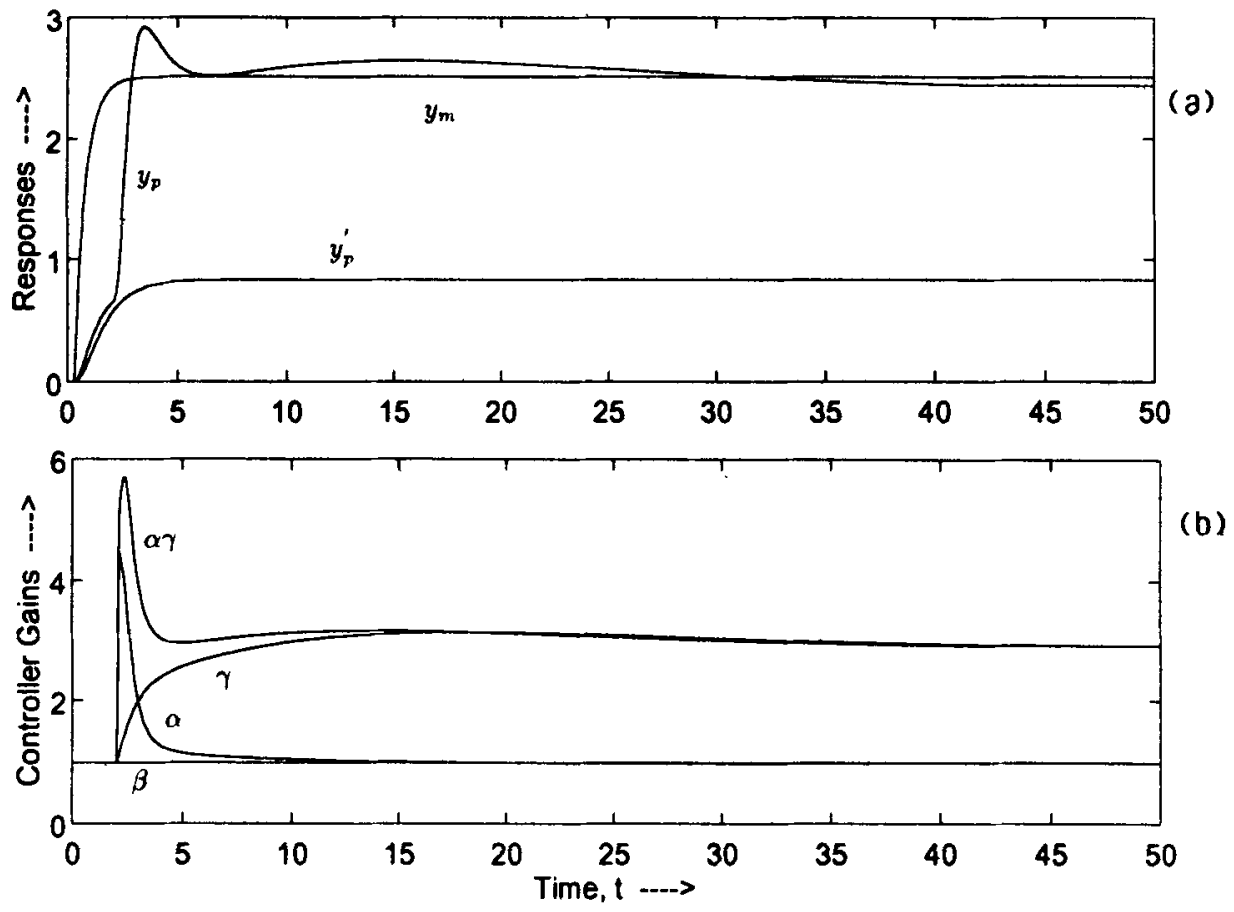

Figure 7. (a) Reference model and plant responses, and (b) controller performance for case 6.
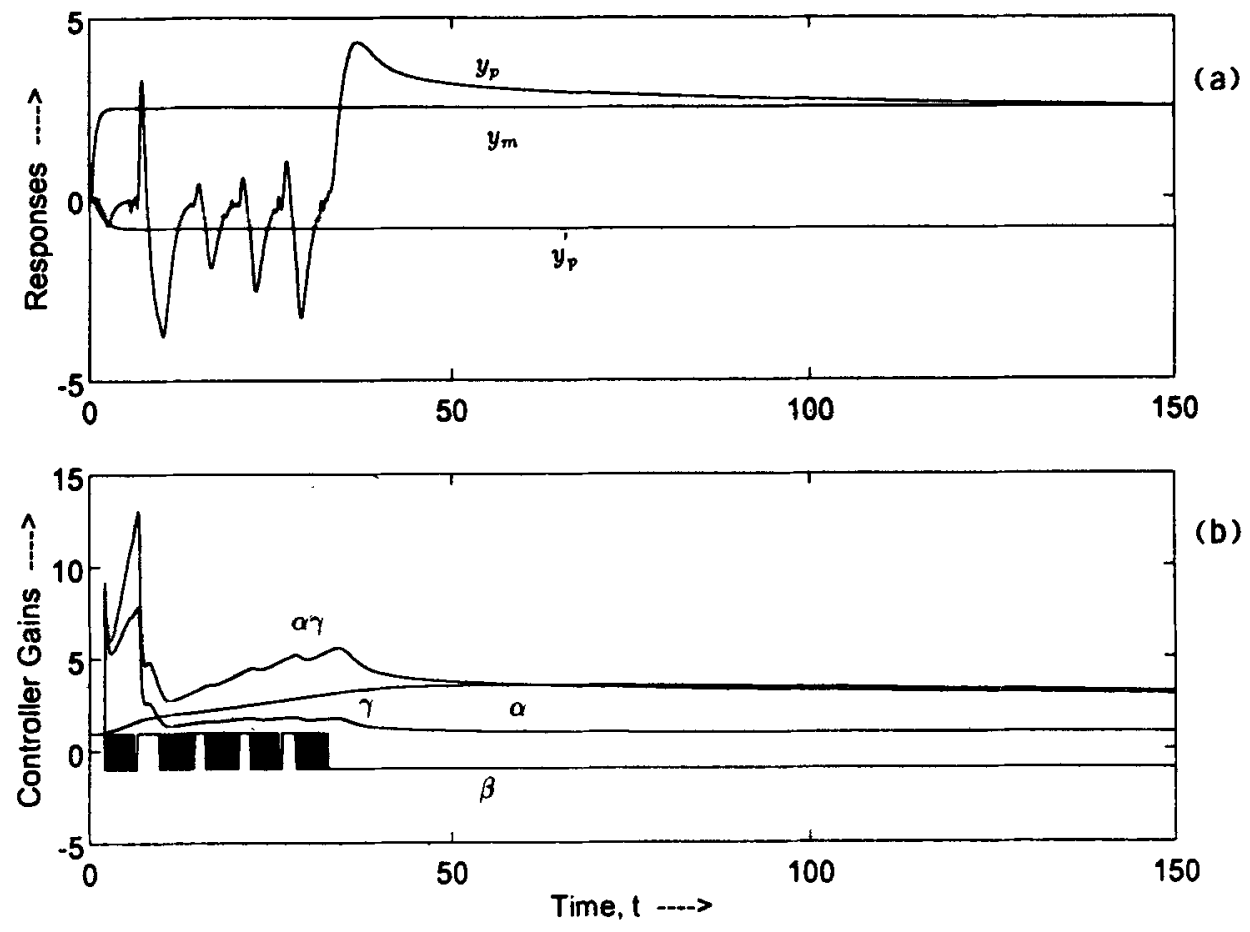

Figure 8. (a) Reference model and plant responses, and (b) controller performance for case 7. 


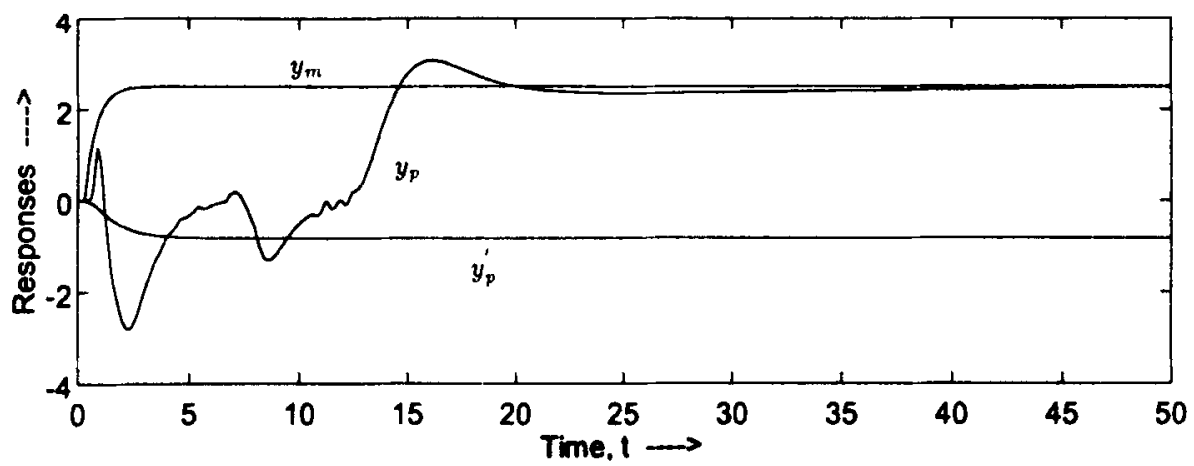

(a)

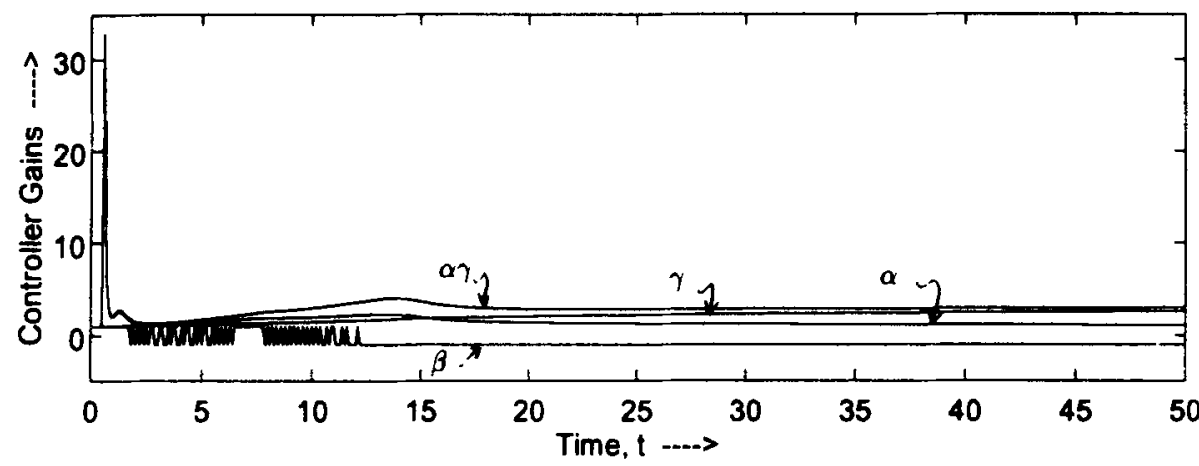

(b)

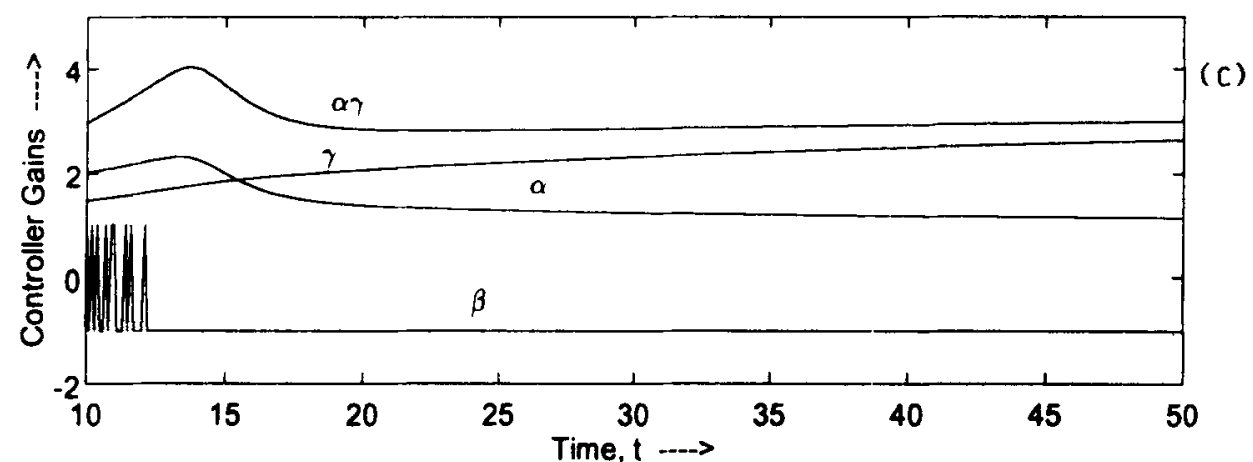

Figure 9. (a) Reference model and plant responses, and (b), (c) controller performance for case 8 . Note the change in the time axis between (b) and (c). 


\section{Conclusion}

In the context of stable MRAC, on relaxing the requirement that the order or an upper bound on the order of the plant be known, and by allowing the plant and the reference model to have nonminimum phase zeros, restriction on the permissible model inputs arises as a natural consequence. Persistency of excitation is done away with. For inputs from a class of 'step-like' functions, the problem is readily formulated in an optimal control framework. Using sub-optimal schemes which are proposed in the beginning as building blocks, two on-line adaptive optimal schemes are designed. The schemes ensure boundedness of the controller and its asymptotic stability.

This paper improves upon the $\varepsilon$-optimal schemes reported in an earlier work (Shankar 1993) for a class of adaptive control problems only recently addressed. The striking features of the algorithms proposed here are simplicity and total time-domain implementation.

The foregoing schemes are attractive in the context of enhanced performance in position control problems where (i) a reduced-order model is often inadequate, and (ii) the parameters of the transfer function vary with the operating conditions. MRAC has been tried out in connection with aircraft control, in power systems and for automatic steering of ships (van Amerongen \& Udnik Ten Cate 1975; Arie et al 1986). This work is a first step towards applications such as autopilots for ships and positioning systems of missile launchers.

For inputs belonging to a class of 'sinusoid-like' functions without and with dc offset, adaptive laws have been designed and similar optimal tuning achieved.

This paper is dedicated to Dr R M Umesh.

The first author wishes to thank Prof. M A L Thathachar for many useful discussions. Dr V Jayashankar, G Ramasubramanian, M T Arvind and K Krishna have kindly extended their help. Our thanks to them.

\section{References}

Arie T, Itoh M, Senoh A, Takahashi N, Fuji S, Misuno N 1986 An adaptive steering system for a ship. IEEE Contr. Syst. Mag. 6: 3-8

Astrom K J 1980 Direct methods for nonminimum phase systems. In Proc. IEEE Conf Decision and Control, Albuquerque, New Mexico, pp 611-615

Astrom K J 1987 Adaptive feedback control. Proc. IEEE 75: 185-217

Bar-Kana I, Kaufman H, Balas M J 1983 Model reference adaptive control of large structural systems. AIAA J. Guid. Contr. 6: 112-118

Benes V E 1965 A nonlinear integral equation in the Marcinkiewicz space $M_{2}$. J. Math. Phys. 44: 24-35

Chen B M, Saberi A, Sannuti P 1992 Necessary and sufficient conditions for a nonminimum phase plant to have a recoverable target loop - A stable compensator design for LTR. Automatica 28: 493-507

Doyle J C, Stein G 1979 Robustness with observers. IEEE Trans. Autom. Contr. 24: 607-611 
Doyle J C, Stein G 1981 Multivariable feedback design: Concepts for a classical/modern synthesis. IEEE Trans. Autom. Contr. 26: 4-16

Goodwin G C, Johnson C R, Sin K S 1981 Global convergence for adaptive one-step ahead optimal controllers based on input matching. IEEE Trans. Autom. Contr. 26: 1269-1273

Hartley T T, Sarantopoulos A D 1991 Using feedback for near one-step-ahead control of inversely unstable plants. IEEE Trans. Autom. Contr. 36: 625-627

Ljung L 1987 System identification: A theory for the user (Englewood Cliffs, NJ: Prentice Hall)

Morse A S 1987 A 4 $(n+1)$-dimensional model reference adaptive system for any relative degree one or two, minimum phase system of dimension $n$ or less. Automatica 23: 123-125

Narendra K S, Annaswamy A M 1989 Stable adaptive systems (Englewood Cliffs, NJ: Prentice Hall)

Praly L 1984 Towards a globally stable direct adaptive control scheme for not necessarily minimum phase systems. IEEE Trans. Autom. Contr. 29: 946-949

Rohrs C E, Valavani L, Athans M, Stein G 1982 Robustness of adaptive control algorithms in the presence of unmodeled dynamics. In Proc. 21st IEEE Conference on Decision and Control, Florida pp 3-11

Rohrs C E, Valavani L, Athans M, Stein G 1985 Robustness of continuous-time adaptive control algorithms in the presence of unmodeled dynamics. IEEE Trans. Autom. Contr. 30: 881-889

Sastry S, Bodson M 1993 Adaptive control - Stability, convergence and robustness (New Delhi: Prentice Hall India)

Shankar H N 1993 Model reference adaptive control of unknown nonminimum phase plants. MSc(Eng.) thesis, Department of Electrical Engineering, Indian Institute of Science, Bangalore

Sliwa S M 1984 An on-line equivalent system identification scheme for adaptive control. $\mathrm{PhD}$ thesis, Department of Aeronautics and Astronautics, Stanford University, Stanford

Sobel K, Kaufman H 1987 Direct model reference adaptive control for a class of MIMO systems. In Advances in control and dynamic systems (ed.) C T Leondes (New York: Academic Press)

Tao G, Ioannou P A 1993 Model reference adaptive control for plants with unknown relative degree. IEEE Trans. Autom. Contr. 38: 976-982

van Amerongen J, Udnik Ten Cate A J 1975 Model reference adaptive autopilots for ships. Automatica 11: 441-449

Zhang Z, Freudenberg J S 1990 Loop transfer recovery for nonminimum phase plants. IEEE Trans. Autom. Contr. 35: 547-553 